Supporting Information for

\title{
Prediction of Photochemically Produced Reactive Intermediates in Surface Waters via Satellite Remote Sensing
}

Yiling Chen ${ }^{\dagger}$, Raymond M. Hozalski ${ }^{\dagger}$, Leif G. Olmanson ${ }^{\ddagger}$, Benjamin P. Page ${ }^{\S}$, Jacques C. Finlay", Patrick L. Brezonik ${ }^{\dagger}$, William A. Arnold ${ }^{\dagger *}$

${ }^{\dagger}$ Department of Civil, Environmental, and Geo- Engineering, University of Minnesota, 500 Pillsbury Drive SE, Minneapolis, MN, 55455-0116, United States

${ }^{\ddagger}$ Department of Forest Resources, University of Minnesota, 1530 Cleveland Avenue North, St. Paul, MN, 55108-6112

${ }^{\S}$ Water Resources Center, University of Minnesota, 1985 Buford Avenue, St. Paul, MN 55108-6112

"Department of Ecology, Evolution, and Behavior, University of Minnesota, 1987 Upper

Buford Circle, St. Paul, MN, 55108-6097

*Corresponding author:

email: arnol032@umn.edu phone: (612)625-8582

Texts: 7

Figures: 18

Tables: 5 


\section{Text S1: Preparation of stock solutions and analytical standards}

The 2,4,6-trimethylphenol (TMP) stock solution for analytical standards (stock 1) was prepared by dissolving $5 \mu \mathrm{M}$ TMP in a $2 \%$ acetonitrile $(\mathrm{ACN})$ solution in a volumetric flask and further diluted with ultrapure water to the desired concentrations. An aqueous TMP stock solution for spiking into water samples (stock 2) was prepared by mixing solid TMP in ultrapure water and centrifuging the solution for $15 \mathrm{~min}$ to remove any undissolved solids prior to spiking, to reach an approximate concentration of $2.5 \mathrm{mM}$. Using the calibration curve obtained using stock 1, the concentration of TMP was calculated to be $4.81 \mu \mathrm{M}$ if $50 \mu \mathrm{L}$ aliquot of stock 2 was spiked into aliquots of $25 \mathrm{~mL}$ water. As a result, $52 \mu \mathrm{L}$ of stock 2 was added into $25 \mathrm{~mL}$ lake water samples to obtain an initial TMP concentration of $\sim 5 \mu \mathrm{M}$. Similarly, analytical standards of 4-carboxybenzophenone (CBP) were prepared by dissolving $0.1 \mathrm{mM} \mathrm{CBP}$ in $20 \% \mathrm{ACN}$ and subsequently diluting with ultrapure water. A stock solution used to spike CBP into water samples was prepared by dissolving CBP in $10 \mathrm{mM}$ borate buffer (10 mM borax mixed with $10 \mathrm{mM}$ boric acid; $\mathrm{pH}$ adjusted to 8.0 using 36-38\% hydrochloric acid) with a target concentration of $1 \mathrm{mM}$, and undissolved solids were removed from the stock solution by centrifugation for $15 \mathrm{~min}$. A terephthalic acid (TPA) stock solution was prepared by adding solid into ultrapure water to achieve a concentration of $10 \mathrm{mM}$ and heating in water bath until dissolved. The TPA stock was stored in the dark to minimize exposure to light.

\section{Text S2: PPRI probes}

${ }^{3}$ DOM*. TMP was used to measure the apparent quantum yield $\left(\Phi_{a p p,}{ }^{3} D^{*} M^{*}\right.$, mol mol-

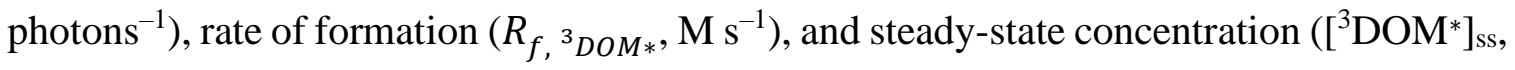


M) of ${ }^{3} \mathrm{DOM}^{*}$. To measure the rate constant for TMP loss $\left(k_{\mathrm{obs}, \mathrm{TMP}}, \mathrm{s}^{-1}\right)$, the desired amount of aqueous TMP stock $2(52 \mu \mathrm{L})$ was added into $25 \mathrm{~mL}$ volumetric flasks and filled to the mark with water sample to reach an initial concentration of $\sim 5 \mu \mathrm{M}$. Previous studies demonstrated that this is the appropriate concentration to measure ${ }^{3} \mathrm{DOM}^{*}$ production from analysis of the pseudo-first order kinetics of TMP. ${ }^{1,2}$ The TMP-containing solutions were irradiated in the solar simulator for 60- to 90 -minute time periods.

Previous studies ${ }^{3-9}$ reported that the second order rate constant for the reaction between ${ }^{3} \mathrm{DOM}^{*}$ and TMP $\left(k^{3}{ }_{D O M * T M P}, \mathrm{M}^{-1} \mathrm{~s}^{-1}\right)$ varies in different natural waters, with a range of $0.81 \times 10^{9}$ to $10 \times 10^{9} \mathrm{M}^{-1} \mathrm{~s}^{-1}$. To obtain $k^{3} D O M *, T M P$, concentrations of [TMP] $]_{0}$ of $50,250,500$ and $750 \mu \mathrm{M}$ were dissolved in a subset of lake samples with varying DOC levels (Island Lake, South Sturgeon Lake, Lake Winnibigosh, Upper Red Lake \#2, and Lake of the Woods (Fourmile Bay)) and stirred continuously for 7 days in the dark. The solutions were then centrifuged for $15 \mathrm{~min}$ to remove undissolved TMP solids prior to irradiation. TMP loss was monitored for 60 to 180 minutes in the solar simulator. The data were fit to equation $\mathrm{S} 1$ to obtain the formation rate of ${ }^{3} \mathrm{DOM}^{*}\left(R_{f},{ }^{3} D O M^{*}\right):^{6}$

$$
\begin{aligned}
& \frac{1}{k_{o b s, T M P}}=\frac{[T M P]_{0}}{R_{f,{ }^{3} D_{O M}}} \\
& +\frac{k_{q}}{R_{f,{ }^{3} D O M *} * k^{3}{ }^{3} D O M^{*}, T M P}
\end{aligned}
$$

where $k_{\mathrm{q}}$ is the pseudo-first order rate constant for all deactivation processes acting on ${ }^{3} \mathrm{DOM}^{*}$. A value of $3.13 \times 10^{5} \mathrm{~s}^{-1}$ was chosen for $k_{\mathrm{q}} \cdot{ }^{4}$ A plot of $1 / k_{\mathrm{obs}, \mathrm{TMP}}$ vs. [TMP] $]_{0}$ yields a slope of $1 / R_{f,}{ }^{3} D O M^{*}$, and $k^{3} D O M^{*}, T M P$ was obtained from the y-intercept. 
The pseudo-first order loss rate constant of TMP ( $\left.k_{\mathrm{obs}, \mathrm{TMP}}\right)$ the product of $k^{3}{ }^{3} O M^{*}, T M P$ and $\left[{ }^{3} \mathrm{DOM}^{*}\right]_{\mathrm{ss} .}{ }^{1,5,10,11}$ Thus, TMP loss is given by equation $\mathrm{S} 2$ :

$\frac{d[T M P]}{d t}=-k_{o b s, T M P}[T M P]=-k^{3}{ }_{D O M^{*}, T M P}[T M P]\left[{ }^{3} D O M^{*}\right]_{S S}$

Additionally, photodegradation of TMP by ${ }^{3} \mathrm{DOM}^{*}$ may be inhibited by the DOC.,12,13 The inhibition factor (IF), for TMP photodegradation was measured using CBP as triplet sensitizer in selected waters. Details are in Text S3.

${ }^{1} \mathbf{O}_{2}$. To obtain the rate of formation $\left(R_{f},{ }^{1} O_{2}, \mathrm{M} \mathrm{s}^{-1}\right)$ and steady-state concentration $\left(\left[{ }^{1} \mathrm{O}_{2}\right]_{\mathrm{ss}}, \mathrm{M}\right)$ of ${ }^{1} \mathrm{O}_{2}$, an aqueous stock solution of $5 \mathrm{mM}$ FFA was added into $25 \mathrm{~mL}$ volumetric flasks and filled with a lake water sample to reach an initial FFA concentration of $20 \mu \mathrm{M} .{ }^{14}$ The solutions were transferred to triplicate quartz tubes and irradiated under the same conditions as the TMP experiments. FFA loss was monitored up to $7 \mathrm{~h}$ in the solar simulator, and data were fit using pseudo-first order kinetics to obtain $k_{\mathrm{obs}, \mathrm{FFA}}\left(\mathrm{s}^{-1}\right)$.

The formation rate of ${ }^{1} \mathrm{O}_{2}\left(R_{f},{ }^{1} O_{2}, \mathrm{M} \mathrm{s}^{-1}\right)$ was computed from:

$\frac{R_{f,{ }^{1} O_{2}}}{k_{o b s, F F A}}=[F F A]_{0}+\frac{k_{d, S}}{k_{S, F F A}}$

where $k_{\mathrm{S}, \mathrm{FFA}}\left(\mathrm{M}^{-1} \mathrm{~s}^{-1}\right)$, the second order rate constant for the reaction between ${ }^{1} \mathrm{O}_{2}$ and FFA, was calculated to be $1.22 \times 10^{8} \mathrm{M}^{-1} \mathrm{~s}^{-1}$ at $30{ }^{\circ} \mathrm{C} .{ }^{15} k_{d, S}\left(\mathrm{~s}^{-1}\right)$ is the pseudo-first order rate constant for the decay of ${ }^{1} \mathrm{O}_{2}$ in water $\left(2.81 \times 10^{5} \mathrm{~s}^{-1}\right) .{ }^{4}$

-OH. $\cdot \mathrm{OH}$ formation was quantified by measuring the production of the fluorescent hydroxyterephthalic acid (hTPA) produced from the reaction of $\bullet \mathrm{OH}$ and $\mathrm{TPA}^{16,17}$ Each $25 \mathrm{~mL}$ volumetric flask of water sample was dosed with $10 \mu \mathrm{M}$ TPA and irradiated up to $8 \mathrm{~h}$ in the solar simulator. Samples were taken periodically, and the formation of hydroxyterephthalic acid (hTPA) by HPLC was monitored to quantify $\bullet \mathrm{OH}$ formation. 


\section{Text S3. Measurement of inhibition factor (IF)}

The reaction rate constants of TMP using pseudo-first order kinetics were obtained in four different solutions: (1) $k_{0}, 10 \mathrm{mM}$ borate buffer at $\mathrm{pH}$ 8; (2) $k_{\mathrm{CBP}}, 10 \mathrm{mM}$ borate buffer at pH 8 with CBP present; (3) $k_{\mathrm{DOM}}$, selected water samples; and (4) $k_{\mathrm{CBP}, \mathrm{DOM} \text {, selected }}$ water samples with CBP present. ${ }^{18}$ Seven samples including Island Lake, South Sturgeon Lake, Lake Vermilion (Pike Bay), Lake Winnibigosh, Upper Red Lake \#2, Lake of the Woods (Fourmile Bay) and Lake Saint Croix were selected out of twenty-four samples as representatives. $k_{0}=0$ because direct photolysis of TMP is not significant. Thus, $k_{\mathrm{CBP}}$ did not need to be corrected for the direct photolysis of TMP. $k_{\mathrm{DOM}}$ was used to correct $k_{\mathrm{CBP}, \mathrm{DOM}}$ to compensate for the ${ }^{3} \mathrm{DOM}^{*}$-induced depletion of TMP. IF was then computed as the ratio of the corrected rate constant with the existence of both CBP and DOM and the corrected rate constant with the existence of CBP only:

$k_{C B P}^{c o r r}=k_{C B P}-k_{0}$

$k_{C B P, D O M}^{c o r r}=k_{C B P, D O M}-k_{D O M}$

$I F=\frac{k_{C B P, D O M}^{c o r r}}{k_{C B P}^{c o r r}}$

It has been reported that 1/IF was linearly dependent on DOC level. A plot of 1/IF versus [DOC] was drawn to calculate the IF values for 24 water samples. The $k_{\text {obs,TMP value for }}$ each sample can then be normalized by its IF to obtain $\mathrm{k}_{\mathrm{obs}, \mathrm{TMP}}^{\mathrm{cor}}$.

Text S4. Determination of Steady-State Concentrations and Quantum Yields of PPRIs

The steady state concentration of ${ }^{3} \mathrm{DOM}^{*}$ is: 
$\left[{ }^{3} D O M^{*}\right]_{S S}=\frac{k_{O b s, T M P}}{k_{{ }^{3} D O M^{*}, T M P}}$

Where $k_{o b s, T M P},\left(\mathrm{~s}^{-1}\right)$, is the rate constant for TMP loss and $k^{{ }^{3} D O M *, T M P}\left(\mathrm{M}^{-1} \mathrm{~s}^{-1}\right)$ is the second order rate constant for reaction between ${ }^{3} \mathrm{DOM}^{*}$ and TMP (see equation S2).

FFA loss was modeled as pseudo-first order reaction, and the steady state concentration of ${ }^{1} \mathrm{O}_{2}$ is:

$\left[{ }^{1} O_{2}\right]_{S S}=\frac{k_{o b s, F F A}}{k_{S, F F A}}$

Where $k_{\text {obs,FFA }}\left(\mathrm{s}^{-1}\right)$ is the rate constant for FFA loss, and $k_{\mathrm{S}, \mathrm{FFA}}\left(\mathrm{M}^{-1} \mathrm{~s}^{-1}\right)$ is the second order rate constant for the reaction between ${ }^{1} \mathrm{O}_{2}$ and FFA.

TPA reaction with $\cdot \mathrm{OH}$ to form hTPA $\left(R_{f, h T P A}, \mathrm{M} \mathrm{s}^{-1}\right)$ follows pseudo-zero order kinetics:

$R_{f, h T P A}=\frac{\Delta[h T P A]}{\Delta t}$

Photodegradation of hTPA in the solar simulator was less than 5\%, and DOM slows its loss via light screening. ${ }^{19}$ Thus, $R_{f, h T P A}$ was not corrected by the direct photolysis rate of hTPA. The steady-state concentration of $\bullet \mathrm{OH}$ was estimated as:

$[\bullet O H]_{S S}=\frac{R_{f, h T P A}}{Y k_{\bullet O H, T P A}[T P A]}$

where $Y$ is the efficiency of hTPA production when oxygen is the oxidant, which is $35 \% ;{ }^{20,21}$ and $k \cdot \mathrm{OH}$,TPA is $4.4 \times 10^{9} \mathrm{M}^{-1} \mathrm{~s}^{-1} \cdot{ }^{16}$ [TPA] was assumed to be constant because TPA loss was minimal. ${ }^{6}$

Under state-state conditions, the rate of formation of $\bullet \mathrm{OH}\left(R_{f} \cdot \mathrm{OH}, \mathrm{M} \mathrm{s}^{-1}\right)$ is equal to its

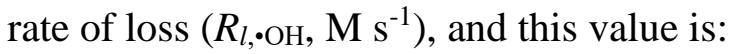

$R_{f, \bullet \mathrm{OH}}=R_{l, \bullet \mathrm{OH}}$ 


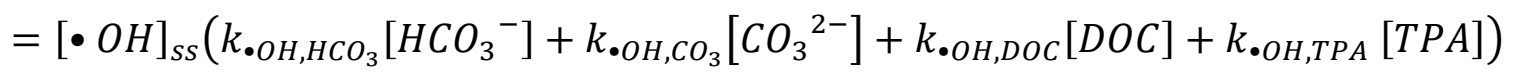

where: $k \cdot \mathrm{OH}, \mathrm{HCO} 3\left(8.5 \times 10^{6} \mathrm{M}^{-1} \mathrm{~s}^{-1}\right), k \cdot \mathrm{OH}, \mathrm{CO} 3\left(3.9 \times 10^{8} \mathrm{M}^{-1} \mathrm{~s}^{-1}\right)$, and $k \cdot \mathrm{OH}, \mathrm{DOC}\left(2.0 \times 10^{4} \mathrm{~L} \mathrm{mgC}^{-}\right.$ $\left.{ }^{1} \mathrm{~s}^{-1}\right)^{22,23}$ are the second order rate constants for the reactions of $\cdot \mathrm{OH}$ with $\mathrm{HCO}_{3}{ }^{-}, \mathrm{CO}_{3}{ }^{2-}$, and DOC, respectively. In addition, halide ions were reported to be scavengers of $\bullet \mathrm{OH}$ in previous studies, ${ }^{24,25}$ especially the $\mathrm{Br}$ ion, which can significant quench $\bullet \mathrm{OH}$, converting - OH to radical reactive halogen species (RHS), leading to ineffective treatment of saline waters. However, in our case, the Br ion should have minor effect on the formation rate of - OH due to its low level in freshwaters.

Quantum yields of the PPRIs $\left(\Phi_{a p p,{ }^{3} D_{O M}}, \Phi_{a p p,{ }^{1} O_{2}}\right.$, and $\Phi_{a p p, \bullet O H}$; mol molphotons $\left.^{-1}\right)$ are defined as the ratio of PPRI formation rate $\left(R_{f}\right)$ to the rate of light absorption by the water sample $\left(R_{a}\right.$, mol-photons $\left.\mathrm{L}^{-1} \mathrm{~s}^{-1}\right)$. Following the method of Sharpless et al., ${ }^{11}$ $R_{a}$ was determined to calculate quantum yields and quantum yield coefficients of PPRIs. A bimolecular actinometer solution of $9.8 \mu \mathrm{M}$ p-nitroanisole/5.5 mM pyridine (PNA/PYD) was used to estimate the spectral irradiance of the $\operatorname{lamp}\left(I_{\lambda}\right.$, mol-photons $\left.\mathrm{L}^{-1} \mathrm{~s}^{-1}\right) \cdot{ }^{6,11} R_{a}$ was calculated from $I_{\lambda}$ :

$R_{a}=\sum_{275 n m}^{600 n m} I_{\lambda}\left(1-10^{-a_{\lambda, d e c}}\right)$

Where $a_{\lambda, \text { dec }}\left(\mathrm{m}^{-1}\right)$ is the decadic absorption coefficients of the water sample for each wavelength; ${ }^{6}$ and $z(\mathrm{~m})$ is the average optical pathlength through the reaction vessels, with a value of $1.12 \times 10^{-2} \mathrm{~m}$ for $13 \times 100 \mathrm{~mm}$ test tubes. ${ }^{26}$ The integration range we chose is from 275 to $600 \mathrm{~nm}$, which captures the full light absorption spectrum of CDOM. ${ }^{27,28}$

The quantum yield coefficient for ${ }^{3} \mathrm{DOM}^{*}\left(f_{\mathrm{TMP}}, \mathrm{L}\right.$ mol-photons $\left.{ }^{-1}\right)$ was calculated by equation S13: 
$f_{T M P}=\frac{k_{o b s, T M P}}{R_{a}}$

The apparent quantum yield of ${ }^{3} \mathrm{DOM}^{*}\left(\Phi_{a p p,}{ }^{3} \mathrm{DOM}^{*}\right.$, mol mol-photons $\left.{ }^{-1}\right)$ was then calculated: ${ }^{6}$

$\Phi_{a p p,{ }^{3} D O M^{*}}=f_{T M P}\left([T M P]_{0}+\frac{k_{q}}{k^{3}{ }_{D O M} M^{*}, T M P}\right)$

The apparent quantum yield of ${ }^{1} \mathrm{O}_{2}\left(\Phi_{a p p,}{ }^{1} O_{2}\right.$, mol mol-photons $\left.{ }^{-1}\right)$ was determined using equation $\mathrm{S} 12$ :

$\Phi_{a p p,{ }^{1} O_{2}}=k_{d, S}$

$$
\times \frac{\left[{ }^{1} O_{2}\right]_{s s}}{R_{a}}
$$

The apparent quantum yield for the formation of $\bullet \mathrm{OH}\left(\Phi_{a p p, \bullet \mathrm{OH}}\right.$, mol mol-photons $\left.{ }^{-1}\right)$ was computed by dividing the rate of loss of $\bullet \mathrm{OH}\left(R_{l, \bullet \mathrm{OH}}, \mathrm{M} \mathrm{s}^{-1}\right)$ by $R_{a}$ :

$$
\begin{aligned}
& \Phi_{a p p, \bullet \mathrm{OH}}=\frac{R_{l, \bullet \mathrm{OH}}}{R_{a}} \\
& =\frac{[\bullet \mathrm{OH}]_{S \mathrm{SS}}\left(k_{\bullet \mathrm{OH}, \mathrm{HCO}}\left[\mathrm{HCO}_{3}{ }^{-}\right]+k_{\bullet \mathrm{OH}, \mathrm{CO}_{3}}\left[\mathrm{CO}_{3}{ }^{2-}\right]+k_{\bullet \mathrm{OH}, \mathrm{DOC}}[\mathrm{DOC}]+k_{\bullet \mathrm{OH}, \mathrm{TPA}}[\mathrm{TPA}]\right)}{R_{a}}
\end{aligned}
$$

Where $k \cdot \mathrm{OH}, \mathrm{HCO} 3\left(8.5 \times 10^{6} \mathrm{M}^{-1} \mathrm{~s}^{-1}\right), k \cdot \mathrm{OH}, \mathrm{CO} 3\left(3.9 \times 10^{8} \mathrm{M}^{-1} \mathrm{~s}^{-1}\right)$, and $k \cdot \mathrm{OH}, \mathrm{DOC}\left(2.0 \times 10^{4} \mathrm{~L}\right.$ $\left.\mathrm{mgC}^{-1} \mathrm{~s}^{-1}\right)^{22,23}$ are the second order rate constants for the reactions of $\bullet \mathrm{OH}$ with $\mathrm{HCO}_{3}$, $\mathrm{CO}_{3}{ }^{2-}$, and DOC, respectively.

\section{Text S5. Correction for DOC-induced inhibition.}

Similar with previous studies, ${ }^{7}$ our results using CBP as a model for ${ }^{3} \mathrm{DOM}^{*}$ demonstrated that the inhibition of TMP photodegradation is likely due to the reduction of 
TMP reaction intermediates. The experimental IF ranged from 0.62 to 1.18 with an average of 1.01. Consistent with previous research, $\mathrm{IF}^{-1}$ linearly correlated with $\mathrm{DOC}\left(\mathrm{R}^{2}=0.93\right.$; Figure S10). To correct for the DOC-induced inhibition, the $k_{\mathrm{obs}, T M P}$ values were normalized to $k_{\text {obs,TMP }}$ corr by IF (eq. S6). Splitting the data into two groups, low to moderately colored waters $\left(a_{440} \leq 11 \mathrm{~m}^{-1}\right)$ and high color waters $\left(a_{440}>11 \mathrm{~m}^{-1}\right)$, an average $6.22 \%$ decrease in $k_{\mathrm{obs}, \mathrm{TMP}}{ }^{\text {corr }}$ for low to moderately colored waters was observed, while $k_{\mathrm{obs}, \mathrm{TMP}}{ }^{\mathrm{corr}}$ of high color waters increased by $65.72 \%$. The average increase in $k_{\mathrm{obs}, \mathrm{TMP}}$ with IF correction was $2.77 \%$ (Figure S5).

The $k_{\mathrm{obs}, \mathrm{TMP}}{ }^{\mathrm{corr}}$ values were used to find $R_{f,{ }^{3}{ }^{3} O M^{*}}$ with a [TMP] $]_{0}$ value of $5 \mu \mathrm{M}$ using equation S1. Experiments measuring $k_{\mathrm{obs}, \mathrm{TMP}}{ }^{\mathrm{corr}}$ as a function of $[\mathrm{TMP}]_{0}$ provided the value of $k_{{ }_{D O M}{ }^{*}, T M P}$ to be $1.72 \times 10^{9} \mathrm{M}^{-1} \mathrm{~s}^{-1}$, similar a reported value of $1.8 \times 10^{9} \mathrm{M}^{-1} \mathrm{~s}^{-1} \cdot{ }^{7}$ Corrected $R_{f,}{ }^{3} D_{O M}{ }^{*}$ were then computed to range from $2.33 \times 10^{-8}$ to $1.75 \times 10^{-7} \mathrm{M} \mathrm{s}^{-1}$ with a mean of $6.41 \times 10^{-8} \mathrm{M} \mathrm{s}^{-1}$ (Table S4).

\section{Text S6. Hypothesis of TMP oxidation by produced ${ }^{3} \mathrm{DOM}$ *}

Because the energy gap between ground state $\mathrm{O}_{2}$ and ${ }^{1} \mathrm{O}_{2}$ is relatively small $\left(94 \mathrm{~kJ} \mathrm{~mol}^{-}\right.$

${ }^{1}$ ), ground state $\mathrm{O}_{2}$ is capable of reacting with both high-energy ${ }^{3} \mathrm{DOM}^{*}\left(>250 \mathrm{~kJ} \mathrm{~mol}^{-1}\right)$ and low-energy ${ }^{3} \mathrm{DOM}^{*}$ (94 to $250 \mathrm{~kJ} \mathrm{~mol}^{-1}$ ) to generate ${ }^{1} \mathrm{O}_{2}$. However, the oneelectron oxidation potential of TMP is $1.22 \mathrm{~V}$, meaning that TMP oxidation by low-energy ${ }^{3} \mathrm{DOM}^{*}(<1.22 \mathrm{~V})$ is thermodynamically unfavorable. Because high-energy ${ }^{3} \mathrm{DOM}^{*}$ only accounts for $15-53 \%$ of the total ${ }^{3} \mathrm{DOM}^{*}$ pool, ${ }^{8}$ TMP samples a smaller fraction of ${ }^{3} \mathrm{CDOM}^{*}$ than ground state $\mathrm{O}_{2}$, leading to a lower value of $\Phi_{a p p,}{ }^{3} D_{0 M} M^{*}$ than 
$\Phi_{a p p,{ }^{1} O_{2}}$ (calculated efficiency $\left.>100 \%\right)$. As stated in the main text, another explantion is different pools of ${ }^{3} \mathrm{DOM} *$ that react via different processes.

\section{Text S7. Models for generating maps.}

The correlations between $R_{a}$, steady-state concentrations and formation rates of PPRIs with $a_{440}$ were used to create lake average level and pixel level maps (Figures 5\&S11-14).

$$
\begin{aligned}
& R_{a}\left(\mathrm{~mol}-\text { photons } L^{-1} \mathrm{~s}^{-1}\right)=1.67 \times 10^{-6} \cdot a_{440}\left(\mathrm{~m}^{-1}\right)+1.77 \times 10^{-6} \\
& R_{f,{ }^{3} \mathrm{DOM}^{*}}\left(\mathrm{M} \mathrm{s}^{-1}\right)=3.75 \times 10^{-9} \cdot a_{440}\left(\mathrm{~m}^{-1}\right)+4.22 \times 10^{-8} \\
& R_{f,{ }^{1} O_{2}}\left(\mathrm{M} \mathrm{s}^{-1}\right)=1.70 \times 10^{-8} \cdot a_{440}\left(\mathrm{~m}^{-1}\right)+7.30 \times 10^{-8} \\
& R_{f, \cdot O H}\left(\mathrm{M} \mathrm{s}{ }^{-1}\right)=3.04 \times 10^{-11} \cdot a_{440}\left(\mathrm{~m}^{-1}\right)+1.12 \times 10^{-11} \\
& {\left[{ }^{3} \mathrm{DOM}{ }^{*}\right]_{S S}(\mathrm{M})=1.17 \times 10^{-14} \cdot a_{440}\left(\mathrm{~m}^{-1}\right)+1.32 \times 10^{-13}} \\
& {\left[{ }^{1} O_{2}\right]_{S S}(\mathrm{M})=5.99 \times 10^{-14} \cdot a_{440}\left(\mathrm{~m}^{-1}\right)+2.58 \times 10^{-13}} \\
& {[\bullet O H]_{S S}(\mathrm{M})=2.69 \times 10^{-16} \cdot \ln a_{440}\left(m^{-1}\right)+1.79 \times 10^{-16}}
\end{aligned}
$$

The estimated first-order rate constants for reactions of diuron and propiconazole were obtained following the equations of Zeng et.al 2013:19

$$
\begin{aligned}
& k_{\text {obs,diuron }}\left(s^{-1}\right)=7.8 \times 10^{9} \cdot\left[{ }^{3} \mathrm{DOM}^{*}\right]_{S S}(\mathrm{M}) \\
&= 7.8 \times 10^{9} \cdot\left(1.17 \times 10^{-14} \cdot a_{440}\left(\mathrm{~m}^{-1}\right)+1.32 \times 10^{-13}\right) \\
& k_{\text {obs,propiconazole }}\left(\mathrm{s}^{-1}\right)=3.9 \times 10^{6} \cdot\left[{ }^{1} \mathrm{O}_{2}\right]_{S S}(\mathrm{M}) \\
&=3.9 \times 10^{6} \cdot\left(5.99 \times 10^{-14} \cdot a_{440}\left(\mathrm{~m}^{-1}\right)+2.58 \times 10^{-13}\right)
\end{aligned}
$$

Thus, 
$k_{\text {obs,diuron }}\left(s^{-1}\right)=9.13 \times 10^{-5} \cdot a_{440}\left(m^{-1}\right)+1.03 \times 10^{-3}$

$k_{\text {obs,propiconazole }}\left(\mathrm{s}^{-1}\right)=2.34 \times 10^{-7} \cdot a_{440}\left(\mathrm{~m}^{-1}\right)+1.01 \times 10^{-6}$

The half-lives were calculated based on the rate law for a first-order reaction,

$$
\begin{aligned}
& t_{1 / 2, \text { diuron }}(s)=0.693 / k_{\text {obs,diuron }}\left(s^{-1}\right) \\
&= \frac{0.693}{9.13 \times 10^{-5} \cdot a_{440}\left(\mathrm{~m}^{-1}\right)+1.03 \times 10^{-3}} \\
&= \frac{1}{1.32 \times 10^{-4} \cdot a_{440}\left(\mathrm{~m}^{-1}\right)+1.49 \times 10^{-3}} \\
& t_{1 / 2, \text { propiconazole }}(s)=0.693 / k_{\text {obs,propiconazole }}\left(\mathrm{s}^{-1}\right) \\
&=\frac{0.693}{2.34 \times 10^{-7} \cdot a_{440}\left(\mathrm{~m}^{-1}\right)+1.01 \times 10^{-6}} \\
&=\frac{1}{3.38 \times 10^{-7} \cdot a_{440}\left(\mathrm{~m}^{-1}\right)+1.46 \times 10^{-6}}
\end{aligned}
$$


Table S1. Chemical suppliers and purities.

\begin{tabular}{cccc} 
Compound & Acronym & Supplier & Purity \\
& & & \\
2,4,6-Trimethylphenol & TMP & Acros Organics & $99 \%$ \\
4-Carboxybenzophenone & $\mathrm{CBP}$ & Sigma-Aldrich & $99 \%$ \\
p-Nitroanisole & $\mathrm{PNA}$ & Acros Organics & $>99 \%$ \\
Pyridine & $\mathrm{PYD}$ & Sigma-Aldrich & $\geq 99 \%$ \\
Furfuryl alcohol & FFA & Sigma-Aldrich & $98 \%$ \\
Terephthalic acid & TPA & Acros Organics & $99 \%$ \\
Hydroxyterephthalic acid & hTPA & TCI & $98 \%$ \\
Boric acid & $\mathrm{H}_{3} \mathrm{BO} 3$ & VWR International & $\geq 99.5 \%$ \\
Phosphoric acid & $\mathrm{H}_{3} \mathrm{PO}_{4}$ & Fisher Scientific & $\geq 85 \% \mathrm{wt}$ \\
Sodium phosphate monobasic & $\mathrm{NaH}_{2} \mathrm{PO}_{4}$ & J.T.Baker & $99 \%$ \\
Sodium phosphate dibasic & $\mathrm{Na}_{2} \mathrm{HPO}_{4}$ & J.T.Baker & $99 \%$ \\
Acetonitrile & $\mathrm{ACN}^{2}$ & Fisher Scientific & $\geq 99.93 \%$ \\
\hline
\end{tabular}

Table S2. HPLC analysis methods for compound detection: 2,4,6-trimethylphenol (TMP), furfuryl alcohol (FFA), hydroxyterephthalic acid (hTPA), and p-nitroanisole (PNA).

\begin{tabular}{|c|c|c|c|c|c|}
\hline Compound & Column & Mobile Phase & $\begin{array}{l}\text { Flow Rate } \\
(\mathrm{mL} / \mathrm{min})\end{array}$ & $\begin{array}{c}\text { Detection } \lambda \\
(\mathbf{n m})^{\mathrm{a}}\end{array}$ & $\begin{array}{l}\text { Retention time } \\
\text { (min) }\end{array}$ \\
\hline TMP & $\begin{array}{l}\text { Ascentis RP-Amide } \\
\text { (Supelco, } 150 \times 4.6 \\
\text { mm, } 5 \mu \mathrm{m} \text { particles) }\end{array}$ & $\begin{array}{l}50 \% \text { Acetonitrile } \\
50 \% 0.1 \% \text { Phosphoric } \\
\text { Acid }\end{array}$ & 1 & $\lambda \mathrm{UV}=205$ & 7.3 \\
\hline FFA & $\begin{array}{l}\text { Ascentis RP-Amide } \\
\text { (Supelco, } 150 \times 4.6 \\
\mathrm{~mm}, 5 \mu \mathrm{m} \text { particles) }\end{array}$ & $100 \%$ Milli-Q $\mathrm{H}_{2} \mathrm{O}$ & 1 & $\lambda \mathrm{UV}=219$ & 5 \\
\hline $\mathrm{TMP}+\mathrm{CBP}$ & $\begin{array}{l}\text { Ascentis RP-Amide } \\
\text { (Supelco, } 150 \times 4.6 \\
\mathrm{~mm}, 5 \mu \mathrm{m} \text { particles) }\end{array}$ & $\begin{array}{l}35 \% \text { Acetonitrile } \\
65 \% \text { Acetate buffer } \mathrm{pH} \\
4.75\end{array}$ & 1 & $\begin{array}{l}\lambda \mathrm{UV}, \mathrm{TMP}= \\
200,254 \\
\lambda_{\mathrm{UV}, \mathrm{CBP}}= \\
265\end{array}$ & $\begin{array}{l}\mathrm{TMP}=10.9 \\
\mathrm{CBP}=3.3\end{array}$ \\
\hline PNA & $\begin{array}{l}\text { Ascentis RP-Amide } \\
\text { (Supelco, } 150 \times 4.6 \\
\text { mm, } 5 \mu \mathrm{m} \text { particles) }\end{array}$ & $\begin{array}{l}60 \% \text { Acetonitrile } \\
40 \% 10 \mathrm{mM} \text { Phosphate } \\
\text { Buffer } \\
\text { (pH 3,10\% Acetonitrile) }\end{array}$ & 1 & $\lambda_{\mathrm{UV}}=313$ & 3.3 \\
\hline hTPA & $\begin{array}{l}\text { Ascentis RP-Amide } \\
\text { (Supelco, } 150 \times 4.6 \\
\mathrm{~mm}, 5 \mu \mathrm{m} \text { particles) }\end{array}$ & $\begin{array}{l}70 \% 10 \mathrm{mM} \text { Phosphate } \\
\text { buffer (pH 2) } \\
30 \% \text { Acetonitrile }\end{array}$ & 1 & $\begin{array}{l}\lambda \mathrm{ex}=312 \\
\lambda \mathrm{em}=428\end{array}$ & 7 \\
\hline
\end{tabular}


Table S3. Water quality and spectral parameters measured for the collected water samples.

\begin{tabular}{|c|c|c|c|c|c|c|c|c|c|c|c|c|}
\hline Site Name & Ecoregion & Latitude & Longitude & pH & $\begin{array}{c}a_{440} \\
\left(\mathrm{~m}^{-1}\right)\end{array}$ & $\begin{array}{c}\text { DOC } \\
\left(\mathbf{m g ~ L}^{-1}\right)\end{array}$ & $\begin{array}{c}\text { SUVA254 }_{254} \\
\left(\mathbf{L ~ m g}^{-1} \mathbf{~ m}^{-1}\right)\end{array}$ & $\begin{array}{c}\text { DIC } \\
(\mathrm{mg} / \mathrm{L})\end{array}$ & E2/E3 & $\begin{array}{c}\mathrm{NO}_{3}^{-} \\
\left(\mathrm{mg} \mathrm{L}^{-1}\right)^{\mathrm{a}}\end{array}$ & $\begin{array}{l}{\left[\mathrm{HCO}_{3}^{-}\right]} \\
(\mathrm{M})\end{array}$ & $\begin{array}{c}{\left[\mathrm{CO}_{3}{ }^{2-}\right]} \\
(\mathrm{M})\end{array}$ \\
\hline Lake Bemidji & NLF & 47.4700 & -94.8629 & 8.43 & 1.15 & 6.28 & 3.66 & 37.4 & 12.00 & $<0.008$ & $6.11 \mathrm{E}-04$ & 7.58E-06 \\
\hline Blandin Reservoir & NLF & 47.2369 & -93.5668 & & 3.68 & 11.0 & 3.18 & 29.6 & 6.29 & 0.057 & & \\
\hline Burntside Lake & NLF & 47.9483 & -91.9442 & 8.76 & 0.78 & 5.49 & 1.99 & 2.09 & 8.31 & $<0.008$ & $3.37 \mathrm{E}-05$ & $8.95 \mathrm{E}-07$ \\
\hline Gull Lake & NLF & 46.4236 & -94.3602 & 7.76 & & 5.97 & & 23.1 & & $<0.008$ & $3.70 \mathrm{E}-04$ & $9.81 \mathrm{E}-07$ \\
\hline Island Lake & NLF & 46.9356 & -95.5224 & & 0.78 & 6.51 & 1.97 & 29.3 & 9.11 & $<0.008$ & & \\
\hline Lake Itasca & NLF & 47.2149 & -95.182 & 8.31 & 0.41 & 6.54 & 1.34 & 35.4 & 10.56 & $<0.008$ & $5.79 \mathrm{E}-04$ & $5.45 \mathrm{E}-06$ \\
\hline Mille Lacs Lake & NLF & 46.1541 & -93.4842 & 7.49 & 2.53 & 6.03 & 1.61 & 15.1 & 5.32 & $<0.008$ & $2.34 \mathrm{E}-04$ & 3.34E-07 \\
\hline Rainy Lake & NLF & 48.6166 & -93.3481 & 7.79 & 4.15 & 10.1 & 3.18 & 3.41 & 5.82 & $<0.008$ & $5.47 \mathrm{E}-05$ & $1.55 \mathrm{E}-07$ \\
\hline Shagawa Lake & NLF & 47.9240 & -91.9133 & 7.57 & 1.70 & 6.76 & 2.87 & 5.77 & 6.74 & $<0.008$ & $9.06 \mathrm{E}-05$ & $1.55 \mathrm{E}-07$ \\
\hline South Sturgeon Lake & NLF & 47.6369 & -93.0645 & 7.96 & 27.9 & 36.2 & 4.85 & 2.73 & 4.53 & 0.079 & 4.42E-05 & $1.86 \mathrm{E}-07$ \\
\hline Sturgeon Lake & NLF & 47.6696 & -93.0565 & & 2.30 & 8.46 & 2.26 & 6.32 & 7.30 & $<0.008$ & $9.52 \mathrm{E}-05$ & $9.39 \mathrm{E}-08$ \\
\hline $\begin{array}{l}\text { Lake Vermilion (Big } \\
\text { Bay) }\end{array}$ & NLF & 47.8473 & -92.3078 & 7.34 & 3.22 & 12.0 & 3.05 & 7.48 & 6.77 & $<0.008$ & $1.13 \mathrm{E}-04$ & $1.14 \mathrm{E}-07$ \\
\hline $\begin{array}{l}\text { Lake Vermilion (Pike } \\
\text { Bay) }\end{array}$ & NLF & 47.8224 & -92.308 & 7.41 & 24.2 & 28.9 & 5.05 & 9.96 & 4.46 & 0.070 & $1.52 \mathrm{E}-04$ & $1.81 \mathrm{E}-07$ \\
\hline White Iron Lake - South & NLF & 47.8608 & -91.8173 & 7.77 & 8.75 & 14.3 & 4.63 & 3.43 & 5.01 & $<0.008$ & $5.49 \mathrm{E}-05$ & $1.49 \mathrm{E}-07$ \\
\hline Lake Winnibigosh & NLF & 47.4285 & -94.057 & 8.47 & 0.92 & 7.37 & 1.79 & 28.6 & 10.62 & $<0.008$ & $4.66 \mathrm{E}-04$ & $6.35 \mathrm{E}-06$ \\
\hline Upper Red Lake \#1 & NMW & 48.1711 & -94.5409 & 8.58 & & 13.4 & & 27.3 & & $<0.008$ & $4.44 \mathrm{E}-04$ & 7.79E-06 \\
\hline Upper Red Lake \#2 & NMW & 48.1743 & -94.5173 & 7.09 & 27.2 & 47.6 & 4.2 & 19.1 & 5.04 & 0.079 & $2.69 \mathrm{E}-04$ & $1.52 \mathrm{E}-07$ \\
\hline $\begin{array}{l}\text { Lake of the Woods } \\
\text { (Muskeg Bay) }\end{array}$ & NMW & 48.9940 & -95.0627 & 7.53 & 5.30 & 11.6 & 3.26 & 9.28 & 5.65 & $<0.008$ & $1.45 \mathrm{E}-04$ & $2.26 \mathrm{E}-07$ \\
\hline $\begin{array}{l}\text { Lake of the Woods } \\
\text { (Fourmile Bay) }\end{array}$ & NMW & 48.8506 & -94.6974 & 8.62 & 6.68 & 13.7 & 3.85 & 6.55 & 5.40 & $<0.008$ & $1.07 \mathrm{E}-04$ & $2.05 \mathrm{E}-06$ \\
\hline Crystal Lake & $\mathrm{NCHF}$ & 46.6241 & -95.9611 & 8.74 & 0.51 & 6.33 & 1.61 & 41.1 & 11.78 & $<0.008$ & $6.65 \mathrm{E}-04$ & $1.69 \mathrm{E}-05$ \\
\hline Detroit Lake & $\mathrm{NCHF}$ & 46.7764 & -95.8403 & 8.64 & 0.83 & 7.02 & 2.1 & 40.2 & 10.24 & 0.106 & $6.53 \mathrm{E}-04$ & $1.32 \mathrm{E}-05$ \\
\hline Mississippi River & $\mathrm{NCHF}$ & 45.0434 & -93.2817 & 7.73 & 2.99 & 9.35 & 3.32 & 47.7 & 6.21 & 1.368 & 7.61E-04 & $1.89 \mathrm{E}-06$ \\
\hline Vadnais Lake & $\mathrm{NCHF}$ & 45.0517 & -93.0937 & 7.94 & 1.84 & 11.3 & 2.36 & 31.4 & 7.69 & 0.138 & 5.07E-04 & 2.04E-06 \\
\hline Lake Saint Croix & WCBP & 44.7499 & -92.8086 & 8.08 & 5.07 & 10.0 & 4.26 & 18.7 & 5.18 & 0.652 & $3.05 \mathrm{E}-04$ & $1.69 \mathrm{E}-06$ \\
\hline
\end{tabular}


Table S4. A summary of photodegradation kinetics.

\begin{tabular}{|c|c|c|c|c|c|c|c|}
\hline Site Name & $\left.\underset{(\text { mol-photons }}{R_{a}} \mathbf{L}^{-1} \mathbf{s}^{-1}\right)$ & $\begin{array}{c}\text { Kobs,TMP } \\
\left(\mathbf{s}^{-1}\right)\end{array}$ & $\begin{array}{c}f_{\text {TMP }} \\
(\mathrm{L} \text { mol-photons } \\
-1\end{array}$ & $\begin{array}{c}\boldsymbol{R}_{\boldsymbol{f},}{ }^{3} D O M^{*} \\
\left(\mathbf{M ~ s}^{-1}\right)\end{array}$ & $\begin{array}{c}k_{\text {obs,FFA }} \\
\left(\mathbf{s}^{-1}\right)\end{array}$ & $\begin{array}{l}R_{f},{ }^{1} o_{2} \\
\left(M^{-1}\right)\end{array}$ & $\begin{array}{l}R_{f, \bullet O H} \\
\left(\mathrm{M} \mathrm{s}^{-1}\right)\end{array}$ \\
\hline Lake Bemidji & 2.32E-06 & $2.40 \mathrm{E}-04$ & $1.03 \mathrm{E}+02$ & 4.49E-08 & $3.17 \mathrm{E}-05$ & 7.36E-08 & $3.99 \mathrm{E}-11$ \\
\hline Blandin Reservoir & 8.74E-06 & $3.62 \mathrm{E}-04$ & $4.15 \mathrm{E}+01$ & $6.76 \mathrm{E}-08$ & $7.50 \mathrm{E}-05$ & $1.74 \mathrm{E}-07$ & \\
\hline Burntside Lake & $3.16 \mathrm{E}-06$ & $1.30 \mathrm{E}-04$ & $4.12 \mathrm{E}+01$ & $2.43 \mathrm{E}-08$ & 2.33E-05 & 5.42E-08 & 0 \\
\hline Gull Lake & 2.67E-06 & 2.22E-04 & $8.33 \mathrm{E}+01$ & 4.15E-08 & $3.17 \mathrm{E}-05$ & 7.36E-08 & $4.16 \mathrm{E}-11$ \\
\hline Island Lake & $2.11 \mathrm{E}-06$ & $1.25 \mathrm{E}-04$ & $5.94 \mathrm{E}+01$ & 2.34E-08 & 2.17E-05 & 5.03E-08 & \\
\hline Lake Itasca & $1.85 \mathrm{E}-06$ & $1.39 \mathrm{E}-04$ & $7.49 \mathrm{E}+01$ & $2.59 \mathrm{E}-08$ & 2.33E-05 & 5.42E-08 & 0 \\
\hline Mille Lacs Lake & 2.01E-06 & $1.25 \mathrm{E}-04$ & $6.22 \mathrm{E}+01$ & 2.33E-08 & $1.67 \mathrm{E}-05$ & 3.87E-08 & 0 \\
\hline Rainy Lake & $8.47 \mathrm{E}-06$ & $3.51 \mathrm{E}-04$ & $4.14 \mathrm{E}+01$ & $6.55 \mathrm{E}-08$ & 5.83E-05 & $1.36 \mathrm{E}-07$ & $1.42 \mathrm{E}-10$ \\
\hline Shagawa Lake & 5.13E-06 & 2.14E-04 & $4.18 \mathrm{E}+01$ & $4.00 \mathrm{E}-08$ & 4.17E-05 & $9.68 \mathrm{E}-08$ & 7.37E-11 \\
\hline South Sturgeon Lake & 4.83E-05 & $4.97 \mathrm{E}-04$ & $1.03 \mathrm{E}+01$ & $9.28 \mathrm{E}-08$ & $1.98 \mathrm{E}-04$ & 4.61E-07 & $4.53 \mathrm{E}-10$ \\
\hline Sturgeon Lake & $4.05 \mathrm{E}-06$ & $2.05 \mathrm{E}-04$ & $5.06 \mathrm{E}+01$ & 3.82E-08 & $3.50 \mathrm{E}-05$ & 8.13E-08 & $6.33 \mathrm{E}-11$ \\
\hline Lake Vermilion (Big Bay) & $8.21 \mathrm{E}-06$ & $3.86 \mathrm{E}-04$ & $4.71 \mathrm{E}+01$ & $7.21 \mathrm{E}-08$ & 7.33E-05 & $1.70 \mathrm{E}-07$ & $1.37 \mathrm{E}-10$ \\
\hline Lake Vermilion (Pike Bay) & $3.95 \mathrm{E}-05$ & 7.87E-04 & $1.99 \mathrm{E}+01$ & $1.47 \mathrm{E}-07$ & $2.05 \mathrm{E}-04$ & 4.76E-07 & $6.90 \mathrm{E}-10$ \\
\hline White Iron Lake - South & $1.95 \mathrm{E}-05$ & 4.16E-04 & $2.13 \mathrm{E}+01$ & 7.77E-08 & $1.00 \mathrm{E}-04$ & 2.32E-07 & $2.88 \mathrm{E}-10$ \\
\hline Lake Winnibigosh & 2.69E-06 & 2.17E-04 & $8.05 \mathrm{E}+01$ & 4.04E-08 & 2.67E-05 & $6.20 \mathrm{E}-08$ & 0 \\
\hline Upper Red Lake \#1 & $3.50 \mathrm{E}-06$ & $3.75 \mathrm{E}-04$ & $1.07 \mathrm{E}+02$ & 7.01E-08 & 4.83E-05 & $1.12 \mathrm{E}-07$ & $6.68 \mathrm{E}-11$ \\
\hline Upper Red Lake \#2 & 4.77E-05 & $9.40 \mathrm{E}-04$ & $1.97 \mathrm{E}+01$ & $1.75 \mathrm{E}-07$ & $2.58 \mathrm{E}-04$ & $6.00 \mathrm{E}-07$ & $1.28 \mathrm{E}-09$ \\
\hline Lake of the Woods (Muskeg Bay) & $9.28 \mathrm{E}-06$ & 3.73E-04 & $4.01 \mathrm{E}+01$ & $6.95 \mathrm{E}-08$ & 7.83E-05 & $1.82 \mathrm{E}-07$ & $1.79 \mathrm{E}-10$ \\
\hline Lake of the Woods (Fourmile Bay) & $1.64 \mathrm{E}-05$ & 4.03E-04 & $2.46 \mathrm{E}+01$ & $7.52 \mathrm{E}-08$ & $8.17 \mathrm{E}-05$ & $1.90 \mathrm{E}-07$ & $2.74 \mathrm{E}-10$ \\
\hline Crystal Lake & $2.22 \mathrm{E}-06$ & $1.62 \mathrm{E}-04$ & $7.31 \mathrm{E}+01$ & 3.03E-08 & 2.33E-05 & $5.42 \mathrm{E}-08$ & $3.05 \mathrm{E}-11$ \\
\hline Detroit Lake & $2.70 \mathrm{E}-06$ & 2.82E-04 & $1.05 \mathrm{E}+02$ & 5.27E-08 & $3.50 \mathrm{E}-05$ & 8.13E-08 & $4.26 \mathrm{E}-11$ \\
\hline Mississippi River & 8.82E-06 & 4.67E-04 & $5.29 \mathrm{E}+01$ & 8.71E-08 & $9.00 \mathrm{E}-05$ & 2.09E-07 & $2.06 \mathrm{E}-10$ \\
\hline Vadnais Lake & $5.67 \mathrm{E}-06$ & 4.01E-04 & $7.08 \mathrm{E}+01$ & 7.49E-08 & $6.83 \mathrm{E}-05$ & $1.59 \mathrm{E}-07$ & 4.10E-11 \\
\hline Lake Saint Croix & 1.19E-05 & 4.27E-04 & $3.59 \mathrm{E}+01$ & $7.96 \mathrm{E}-08$ & 9.67E-05 & $2.25 \mathrm{E}-07$ & $1.92 \mathrm{E}-10$ \\
\hline
\end{tabular}


Table S5. A summary of the steady-state concentrations and quantum yields of PPRIs.

\begin{tabular}{|c|c|c|c|c|c|c|}
\hline Site Name & $\begin{array}{c}{\left[{ }^{3} \mathrm{CDOM}^{*}\right] \mathrm{ss}} \\
\text { (M) }\end{array}$ & $\begin{array}{c}\Phi_{a p p,{ }^{3}{ }^{3} D M^{*}} \\
\left(\mathrm{~mol} \mathrm{mol-photons}^{-1}\right)\end{array}$ & $\begin{array}{c}{\left[{ }^{1} \mathbf{O}_{2}\right] \mathrm{SS}} \\
(\mathbf{M})\end{array}$ & $\begin{array}{c}\Phi_{a p p,{ }^{1} o_{2}} \\
\left(\mathrm{~mol} \mathrm{~mol} \text {-photons }^{-1}\right)\end{array}$ & $\begin{array}{c}{[\cdot \mathbf{O H}] \mathrm{ss}} \\
(\mathrm{M})\end{array}$ & $\begin{array}{c}\Phi_{a p p, \bullet \circ H} \\
\left(\text { mol mol-photons }^{-1}\right)\end{array}$ \\
\hline Lake Bemidji & $1.40 \mathrm{E}-13$ & $1.88 \mathrm{E}-02$ & $2.60 \mathrm{E}-13$ & $3.14 \mathrm{E}-02$ & $2.24 \mathrm{E}-16$ & $1.72 \mathrm{E}-05$ \\
\hline Blandin Reservoir & $2.11 \mathrm{E}-13$ & 7.53E-03 & $6.15 \mathrm{E}-13$ & $1.98 \mathrm{E}-02$ & $5.91 \mathrm{E}-16$ & \\
\hline Burntside Lake & $7.56 \mathrm{E}-14$ & $7.48 \mathrm{E}-03$ & $1.91 \mathrm{E}-13$ & $1.70 \mathrm{E}-02$ & 0 & 0 \\
\hline Gull Lake & $1.29 \mathrm{E}-13$ & $1.51 \mathrm{E}-02$ & $2.60 \mathrm{E}-13$ & $2.73 \mathrm{E}-02$ & $2.49 \mathrm{E}-16$ & $1.56 \mathrm{E}-05$ \\
\hline Island Lake & $7.29 \mathrm{E}-14$ & $1.08 \mathrm{E}-02$ & $1.78 \mathrm{E}-13$ & 2.37E-02 & 0 & \\
\hline Lake Itasca & $8.06 \mathrm{E}-14$ & $1.36 \mathrm{E}-02$ & $1.91 \mathrm{E}-13$ & $2.91 \mathrm{E}-02$ & 0 & 0 \\
\hline Mille Lacs Lake & 7.27E-14 & $1.13 \mathrm{E}-02$ & $1.37 \mathrm{E}-13$ & $1.91 \mathrm{E}-02$ & 0 & 0 \\
\hline Rainy Lake & $2.04 \mathrm{E}-13$ & 7.52E-03 & $4.78 \mathrm{E}-13$ & $1.59 \mathrm{E}-02$ & $5.76 \mathrm{E}-16$ & $1.68 \mathrm{E}-05$ \\
\hline Shagawa Lake & $1.25 \mathrm{E}-13$ & $7.59 \mathrm{E}-03$ & $3.42 \mathrm{E}-13$ & $1.87 \mathrm{E}-02$ & $4.09 \mathrm{E}-16$ & $1.44 \mathrm{E}-05$ \\
\hline South Sturgeon Lake & $2.89 \mathrm{E}-13$ & $1.87 \mathrm{E}-03$ & $1.63 \mathrm{E}-12$ & $9.46 \mathrm{E}-03$ & $5.91 \mathrm{E}-16$ & $9.39 \mathrm{E}-06$ \\
\hline Sturgeon Lake & $1.19 \mathrm{E}-13$ & $9.18 \mathrm{E}-03$ & $2.87 \mathrm{E}-13$ & $1.99 \mathrm{E}-02$ & $2.96 \mathrm{E}-16$ & $1.56 \mathrm{E}-05$ \\
\hline Lake Vermilion (Big Bay) & $2.25 \mathrm{E}-13$ & $8.55 \mathrm{E}-03$ & $6.01 \mathrm{E}-13$ & $2.06 \mathrm{E}-02$ & $4.80 \mathrm{E}-16$ & $1.66 \mathrm{E}-05$ \\
\hline Lake Vermilion (Pike Bay) & 4.57E-13 & $3.62 \mathrm{E}-03$ & $1.68 \mathrm{E}-12$ & $1.20 \mathrm{E}-02$ & $1.11 \mathrm{E}-15$ & $1.75 \mathrm{E}-05$ \\
\hline White Iron Lake - South & $2.42 \mathrm{E}-13$ & $3.88 \mathrm{E}-03$ & $8.20 \mathrm{E}-13$ & $1.18 \mathrm{E}-02$ & $8.70 \mathrm{E}-16$ & $1.48 \mathrm{E}-05$ \\
\hline Lake Winnibigosh & $1.26 \mathrm{E}-13$ & $1.46 \mathrm{E}-02$ & $2.19 \mathrm{E}-13$ & $2.28 \mathrm{E}-02$ & 0 & 0 \\
\hline Upper Red Lake \#1 & $2.18 \mathrm{E}-13$ & $1.95 \mathrm{E}-02$ & $3.96 \mathrm{E}-13$ & $3.18 \mathrm{E}-02$ & $2.10 \mathrm{E}-16$ & $1.91 \mathrm{E}-05$ \\
\hline Upper Red Lake \#2 & $5.46 \mathrm{E}-13$ & $3.58 \mathrm{E}-03$ & $2.12 \mathrm{E}-12$ & $1.25 \mathrm{E}-02$ & $1.28 \mathrm{E}-15$ & $2.68 \mathrm{E}-05$ \\
\hline Lake of the Woods (Muskeg Bay) & $2.17 \mathrm{E}-13$ & 7.29E-03 & $6.42 \mathrm{E}-13$ & $1.94 \mathrm{E}-02$ & $6.46 \mathrm{E}-16$ & $1.93 \mathrm{E}-05$ \\
\hline Lake of the Woods (Fourmile Bay) & $2.34 \mathrm{E}-13$ & $4.46 \mathrm{E}-03$ & $6.69 \mathrm{E}-13$ & $1.15 \mathrm{E}-02$ & $8.59 \mathrm{E}-16$ & $1.67 \mathrm{E}-05$ \\
\hline Crystal Lake & $9.44 \mathrm{E}-14$ & $1.33 \mathrm{E}-02$ & $1.91 \mathrm{E}-13$ & $2.42 \mathrm{E}-02$ & $1.67 \mathrm{E}-16$ & $1.37 \mathrm{E}-05$ \\
\hline Detroit Lake & $1.64 \mathrm{E}-13$ & $1.90 \mathrm{E}-02$ & $2.87 \mathrm{E}-13$ & 2.99E-02 & $2.18 \mathrm{E}-16$ & $1.58 \mathrm{E}-05$ \\
\hline Mississippi River & $2.71 \mathrm{E}-13$ & $9.61 \mathrm{E}-03$ & 7.38E-13 & $2.35 \mathrm{E}-02$ & $8.64 \mathrm{E}-16$ & $2.33 \mathrm{E}-05$ \\
\hline Vadnais Lake & $2.33 \mathrm{E}-13$ & $1.29 \mathrm{E}-02$ & $5.60 \mathrm{E}-13$ & $2.78 \mathrm{E}-02$ & $1.49 \mathrm{E}-16$ & $7.24 \mathrm{E}-06$ \\
\hline Lake Saint Croix & $2.48 \mathrm{E}-13$ & $6.51 \mathrm{E}-03$ & $7.92 \mathrm{E}-13$ & $1.87 \mathrm{E}-02$ & $7.76 \mathrm{E}-16$ & $1.61 \mathrm{E}-05$ \\
\hline
\end{tabular}




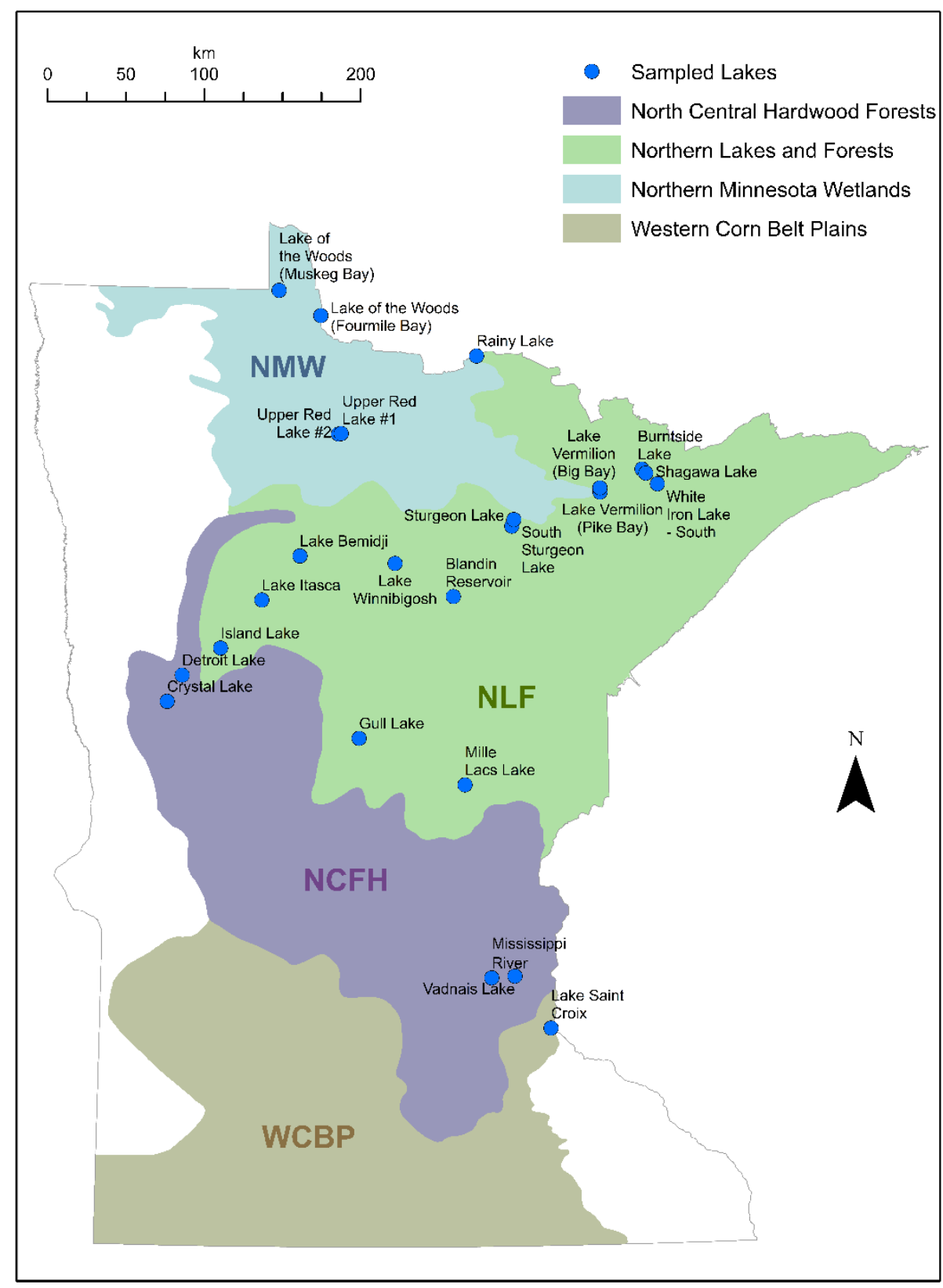

Figure S1. Map showing locations of 24 inland waters sampled throughout Minnesota. 


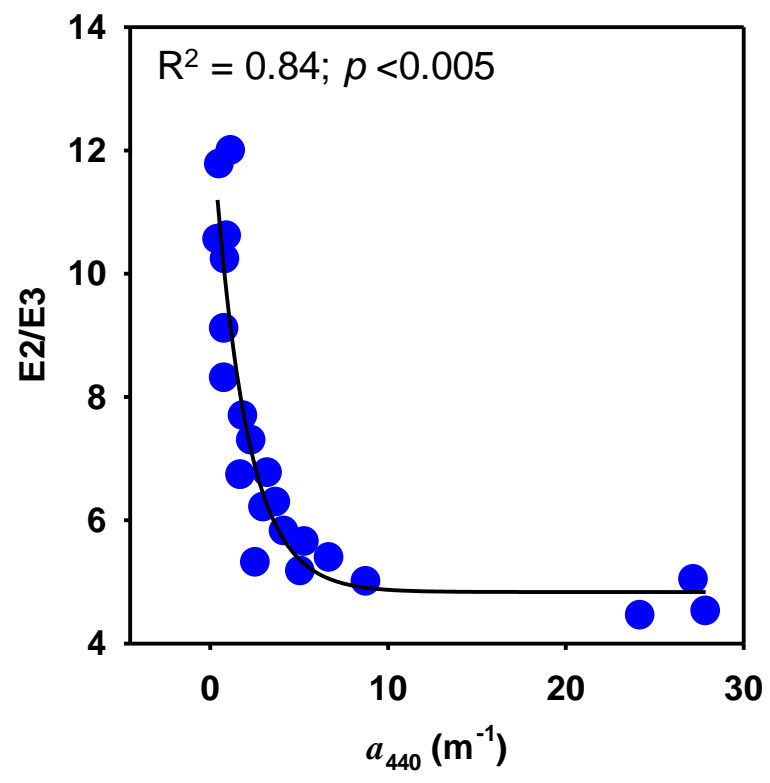

Figure S2. Correlations of E2/E3 with $a_{440}$. The regression equation is E2/E3 = $7.96 \cdot \exp \left(-0.54 \cdot a_{440}\left(\mathrm{~m}^{-1}\right)\right)+4.84$.
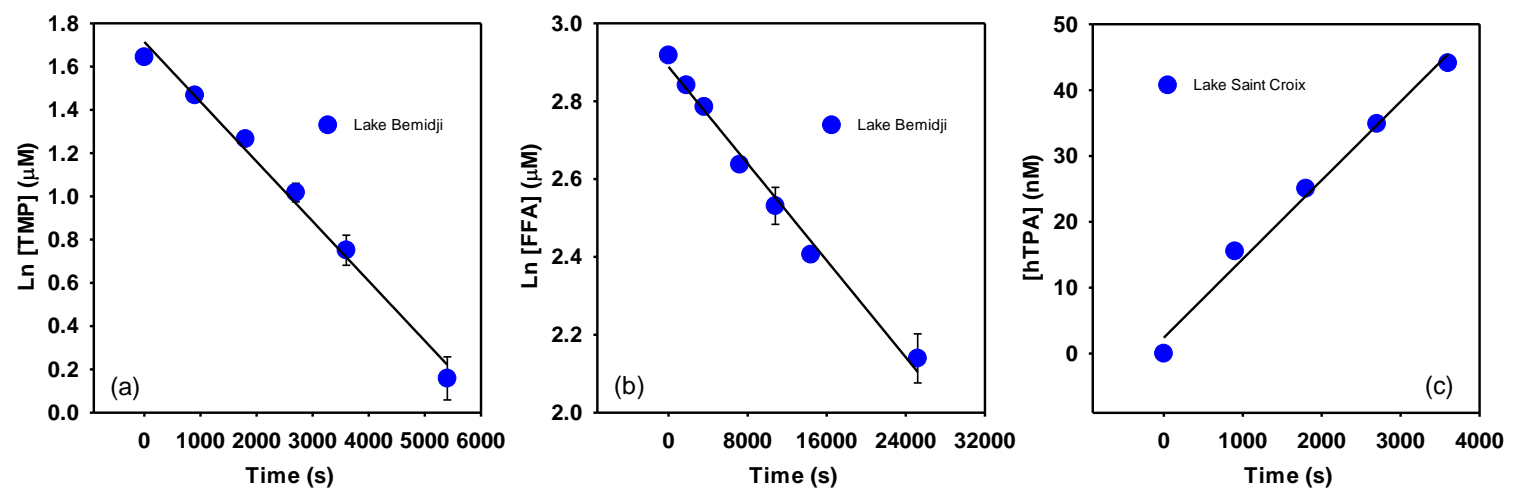

Figure S3. Time course of TMP photodegradation in Lake Bemidji, FFA photodegradation in Lake Bemidji, and hTPA formation in Lake Saint Croix. 

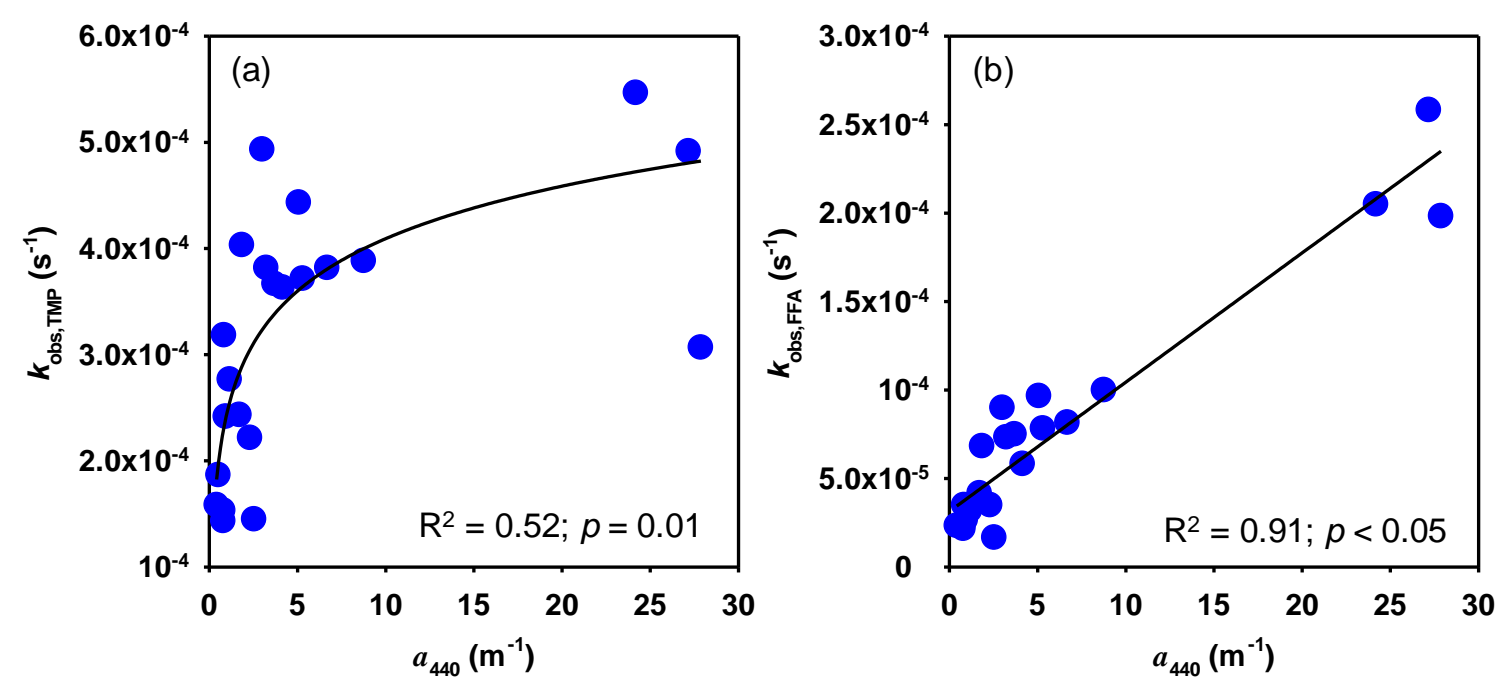

Figure S4. Correlations of $k_{\mathrm{obs}, \mathrm{TMP}}$ and $k_{\mathrm{obs}, \mathrm{FFA}}$ with $a_{440}$. The regression equations are (a) $k_{\text {obs,TMP }}\left(\mathrm{s}^{-1}\right)=0.0043 \cdot a_{440}\left(\mathrm{~m}^{-1}\right)+0.015$; (b) $k_{\text {obs,FFA }}\left(\mathrm{s}^{-1}\right)=0.0004 \cdot a_{440}\left(\mathrm{~m}^{-1}\right)+0.0019$.

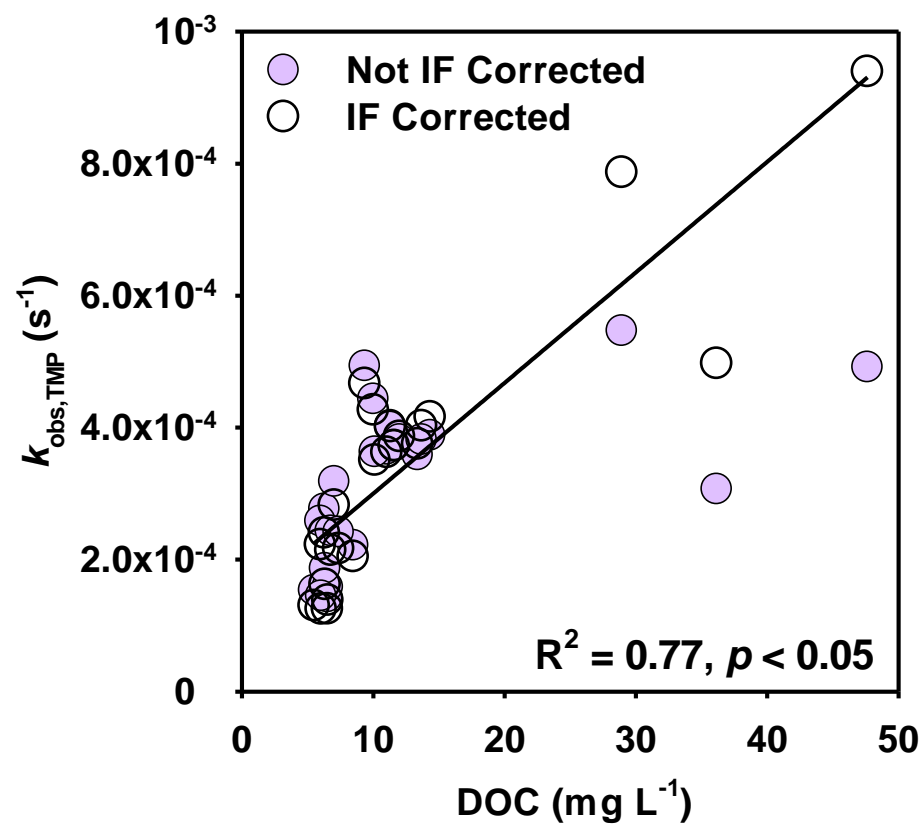

Figure S5. The correlations of $k_{\mathrm{obs}, \mathrm{TMP}}$ versus DOC before and after correction using the inhibition factor, IF. The regression equation after correction is $k_{\mathrm{obs}, \mathrm{TMP}}\left(\mathrm{s}^{-1}\right)=1.68 \times 10^{-}$ $5 \cdot \mathrm{DOC}(\mathrm{mg} / \mathrm{L})+1.98 \times 10^{-6}$. 

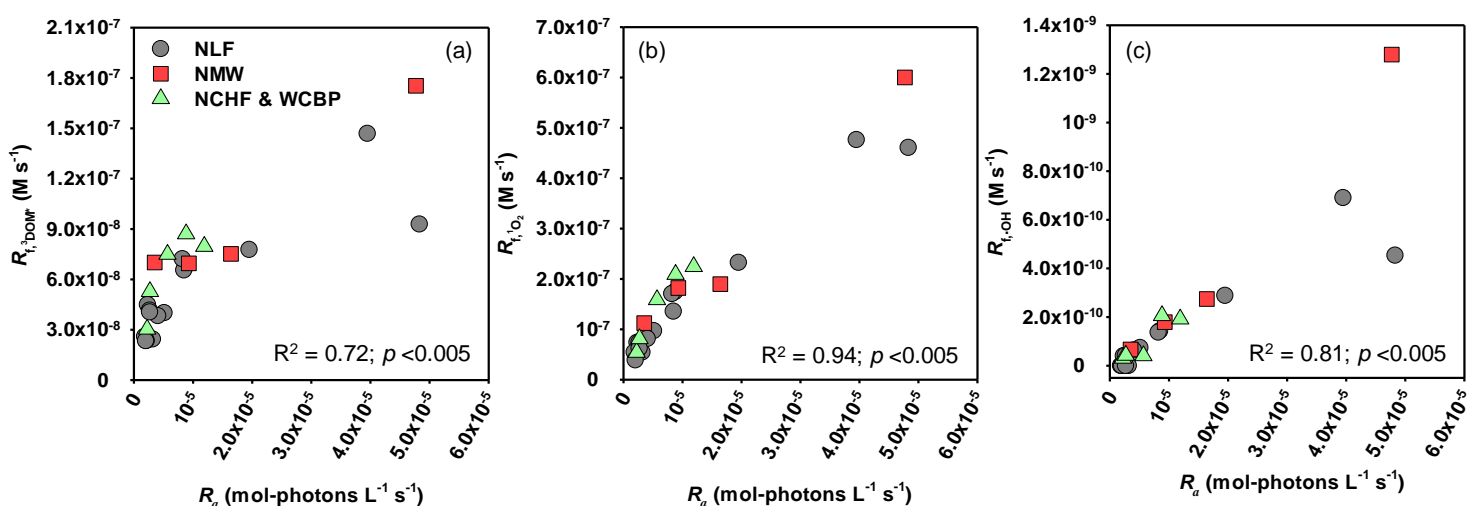

Figure S6. Plots of formation of ${ }^{3} \mathrm{DOM}^{*},{ }^{1} \mathrm{O}_{2}$ and $\cdot \mathrm{OH}$ versus $R_{a}$. The slope from the origin to each point is the (apparent) quantum yield for the given water sample. The small range in slopes implies a small range of (apparent) quantum yields.
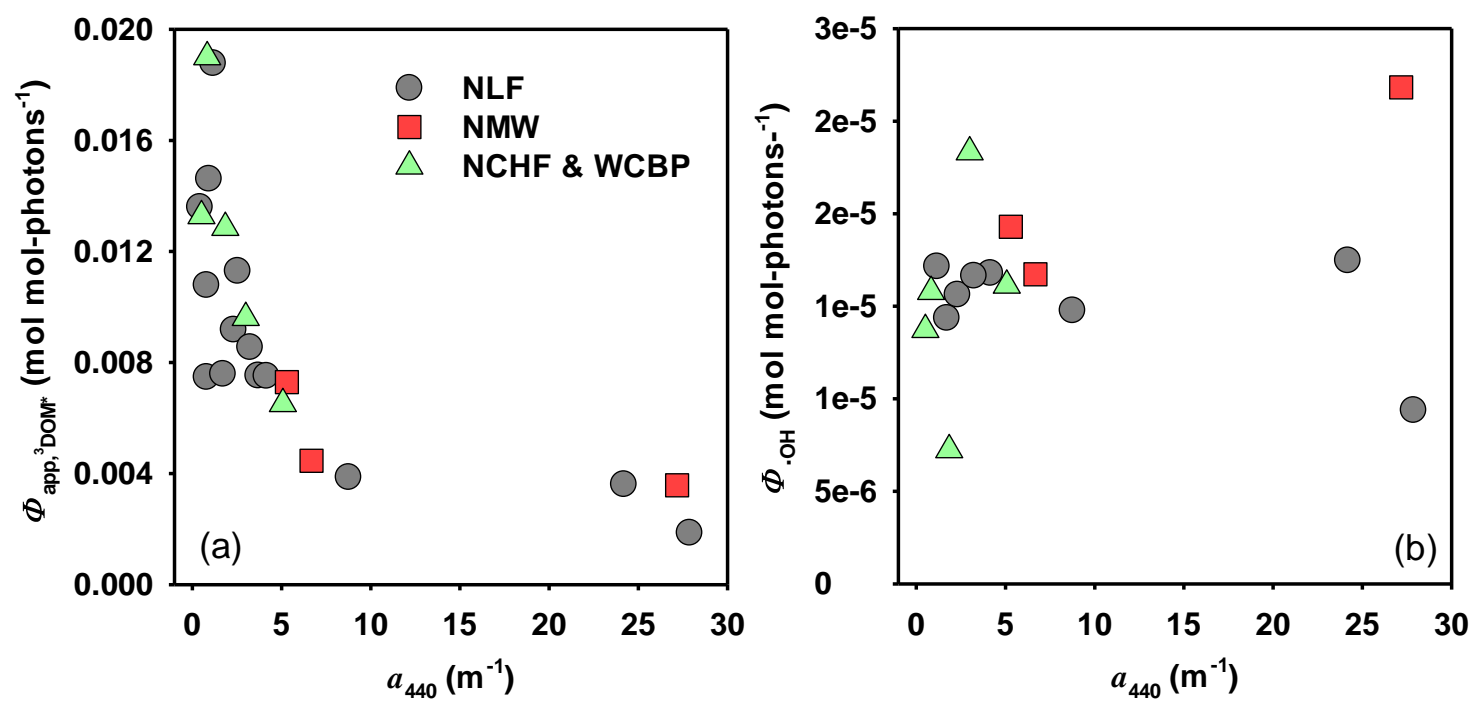

Figure S7. Relationships between (a) $\Phi_{a p p,{ }^{3} D_{O M}}$ and $a_{440}$; (b) $\Phi_{a p p, \bullet O H}$ and $a_{440}$. 


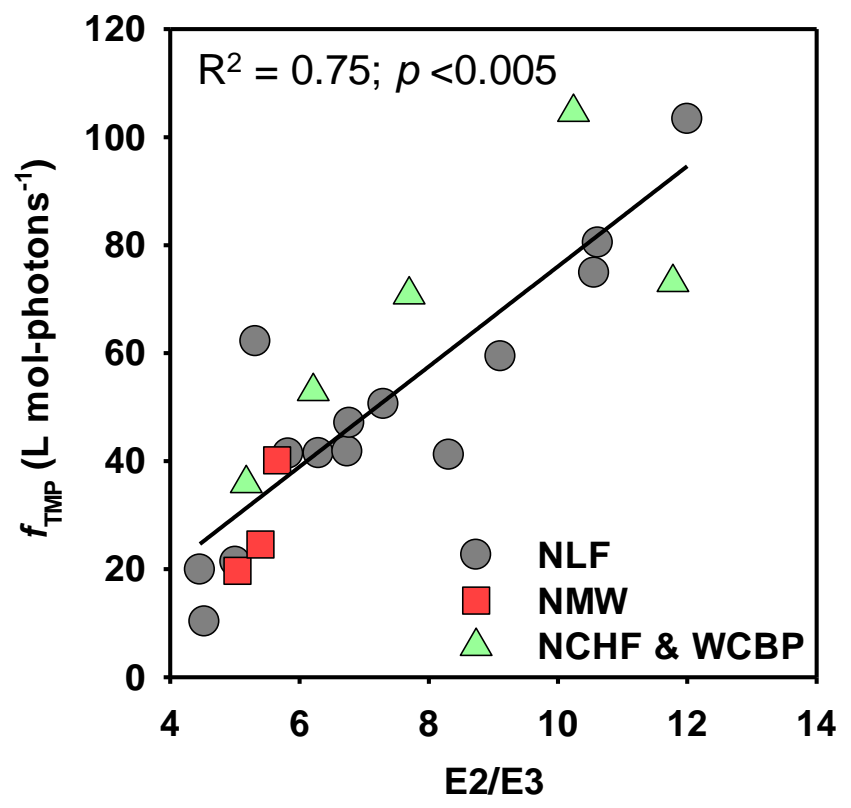

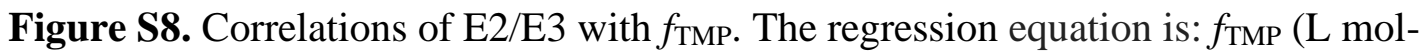
photons $\left.^{-1}\right)=9.28( \pm 1.19) \cdot \mathrm{E} 2 / \mathrm{E} 3-16.7( \pm 9.11)$.
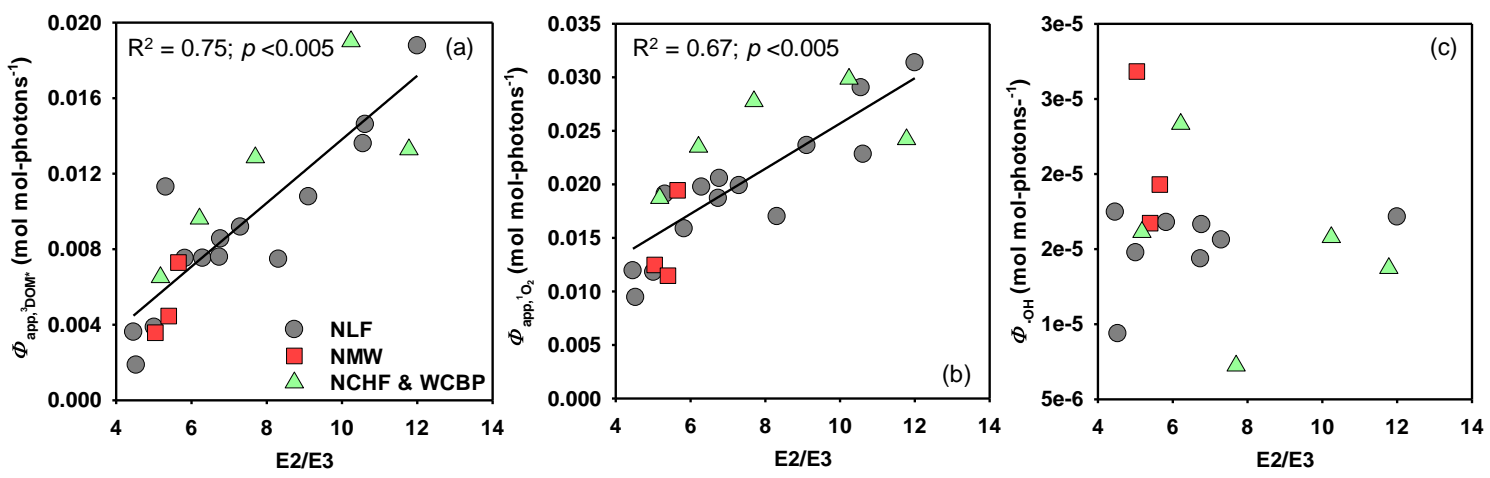

Figure S9. Correlations of E2/E3 with $\Phi_{a p p,}{ }^{3} D_{O M}{ }^{*}$ and $\Phi_{1} O_{2}$. The regression equations

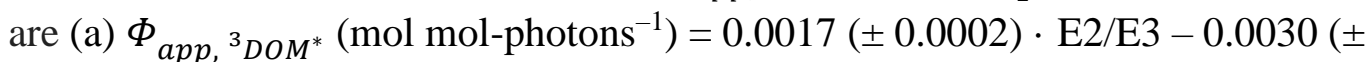
$0.0017)$; (b) $\Phi_{a p p,{ }^{1} O_{2}}\left(\mathrm{~mol} \mathrm{mol-photons}{ }^{-1}\right)=0.0021( \pm 0.0003) \cdot \mathrm{E} 2 / \mathrm{E} 3-0.0046( \pm$ 0.0026). (c) shows the relationship between E2/E3 and $\Phi_{a p p, \bullet O H}$. 

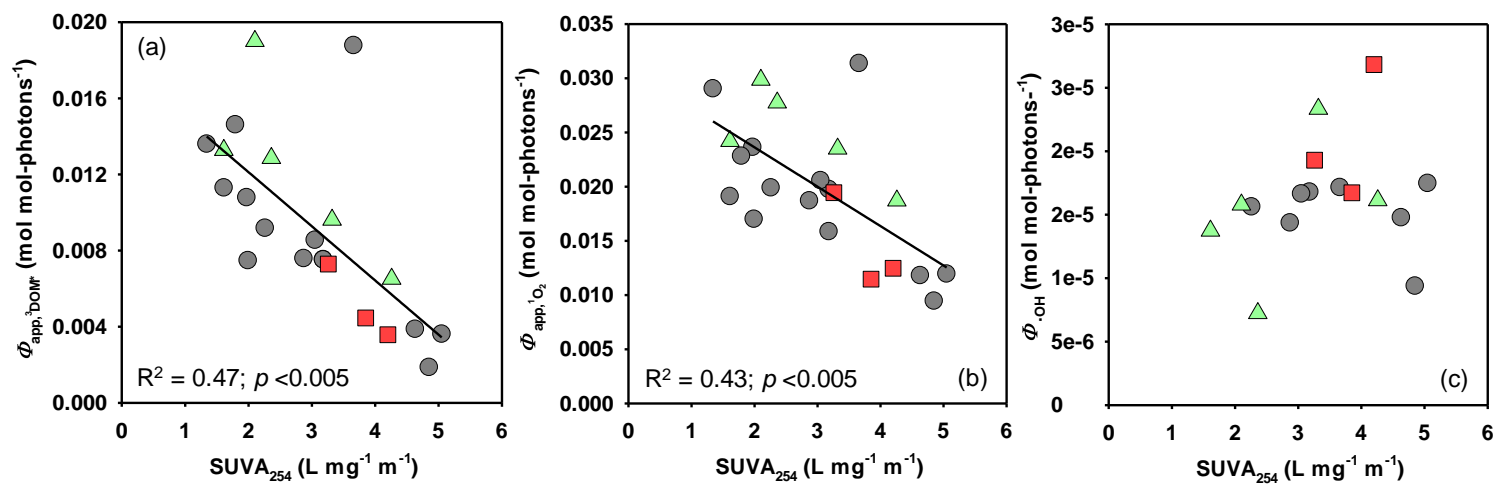

Figure S10. Correlations of SUVA 254 with $\Phi_{a p p,{ }^{3} D O M^{*}}$ and $\Phi_{{ }^{1} O_{2}}$. The regression equations are (a) $\Phi_{a p p,{ }^{3} D_{0 M}}\left(\mathrm{~mol} \mathrm{~mol}-\right.$ photons $\left.^{-1}\right)=-0.0028( \pm 0.0007) \cdot \mathrm{SUVA}_{254}(\mathrm{~L}$ $\left.\mathrm{mg}^{-1} \mathrm{~m}^{-1}\right)+0.0178( \pm 0.0022)$; (b) $\Phi_{a p p,{ }^{1} O_{2}}\left(\mathrm{~mol} \mathrm{~mol}-\right.$ photons $\left.^{-1}\right)=-0.0036( \pm$ $0.0009) \cdot \operatorname{SUVA}_{254}\left(\mathrm{~L} \mathrm{mg}^{-1} \mathrm{~m}^{-1}\right)+0.0309$ ( \pm 0.0030). (c) shows the relationship between $\mathrm{SUVA}_{254}$ and $\Phi_{a p p, \bullet \mathrm{H}}$.

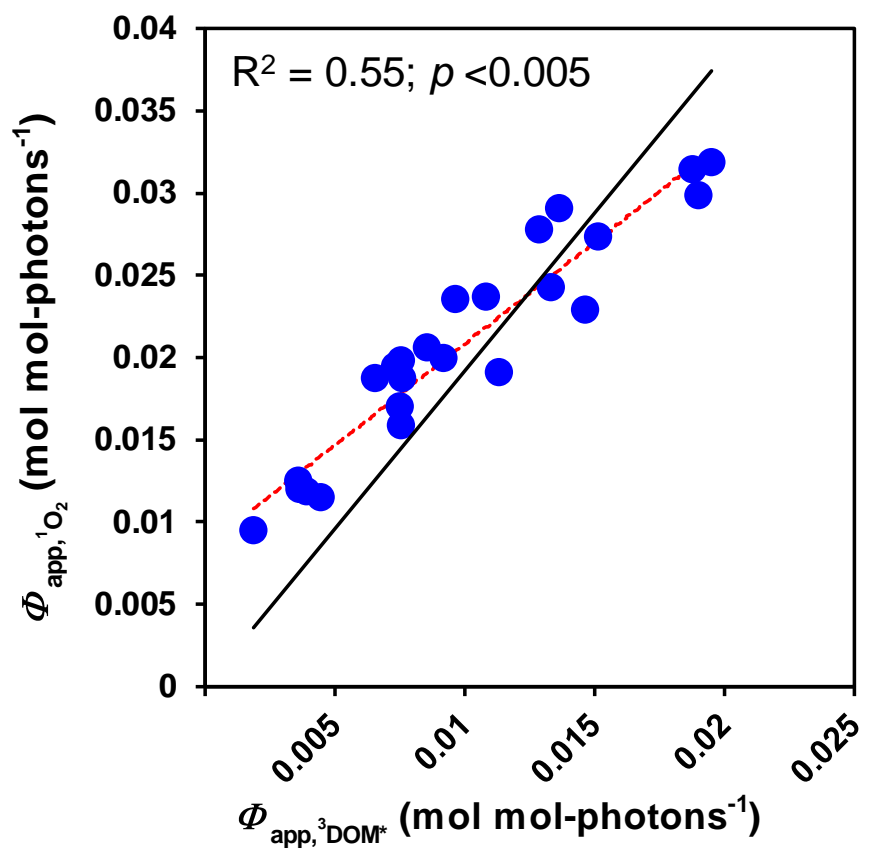

Figure S11. $\Phi_{a p p,{ }^{1} O_{2}}$ versus $\Phi_{a p p,{ }^{3} D O M^{*}}$. The slope of the linear relationship with/without the intercept forced through 0 is 1.92 and 1.23 , respectively. 


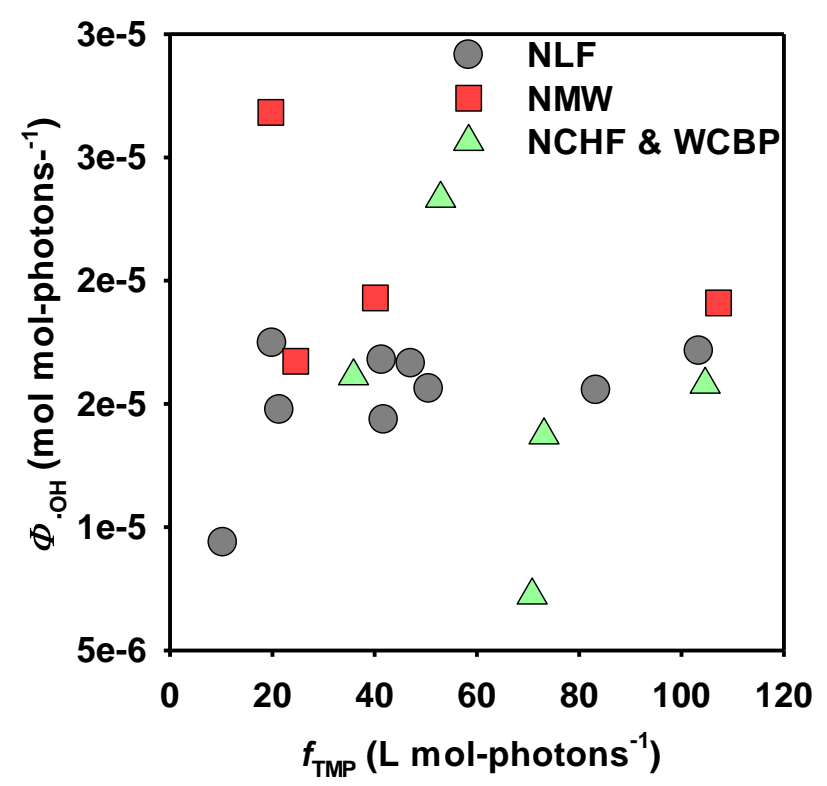

Figure S12. Relationship between $f_{T M P}$ and $\Phi_{a p p, \bullet O H}$.

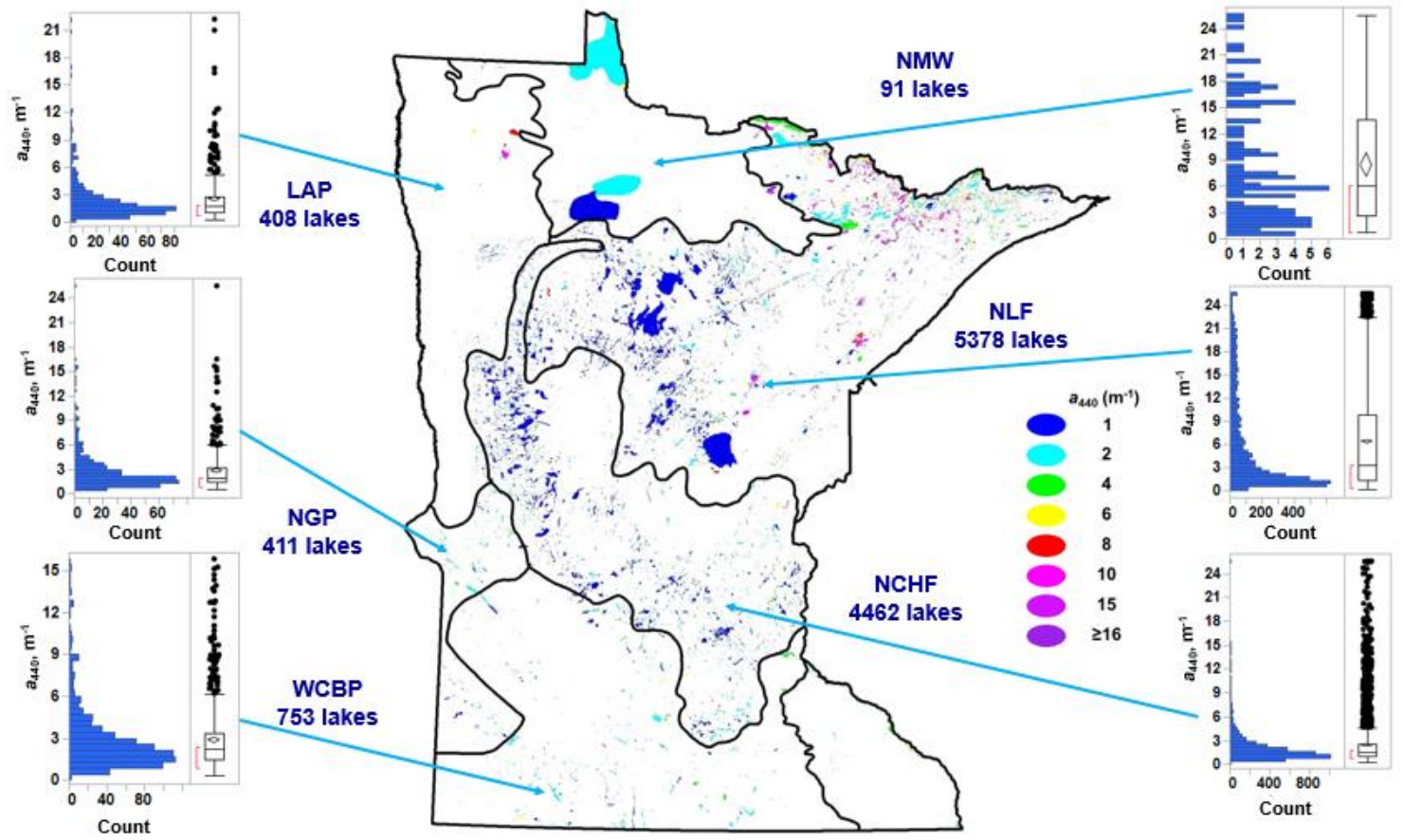

Figure S13. Minnesota 2015 and 2016 mean CDOM distribution for all measured waterbodies by ecoregion. This figure is fromref. ${ }^{29}$ and is reproduced with permission from Elsevier. 


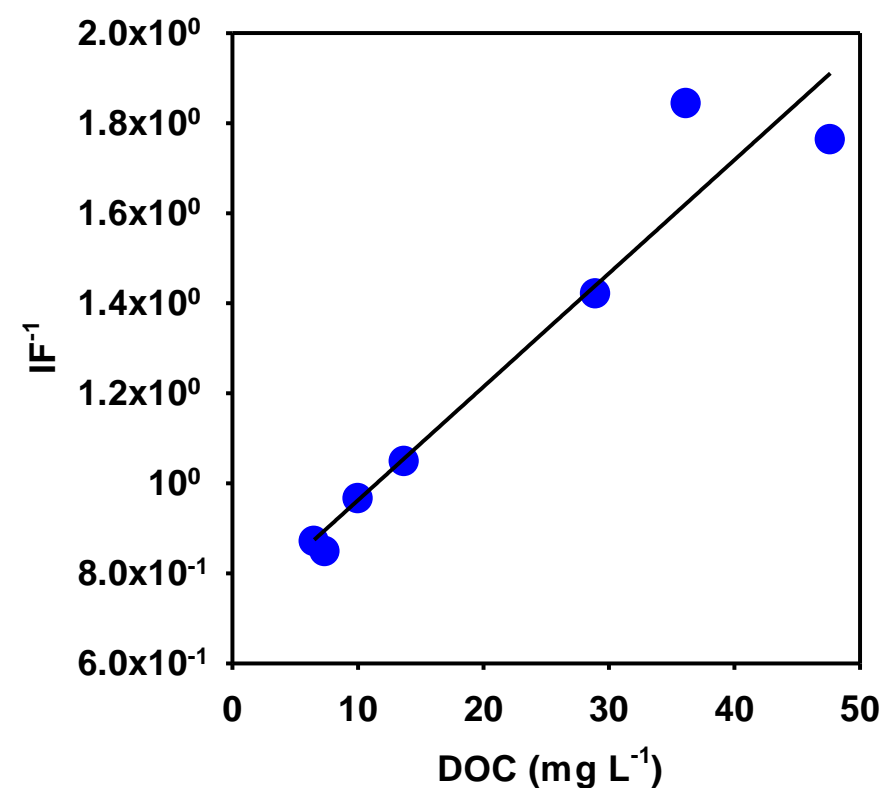

Figure S14. Correlation of $\mathrm{IF}^{-1}$ with DOC. The regression equation is $\mathrm{IF}^{-1}=0.025( \pm$ $0.003) \cdot \operatorname{DOC}(\mathrm{mg} / \mathrm{L})+0.71( \pm 0.08)$.

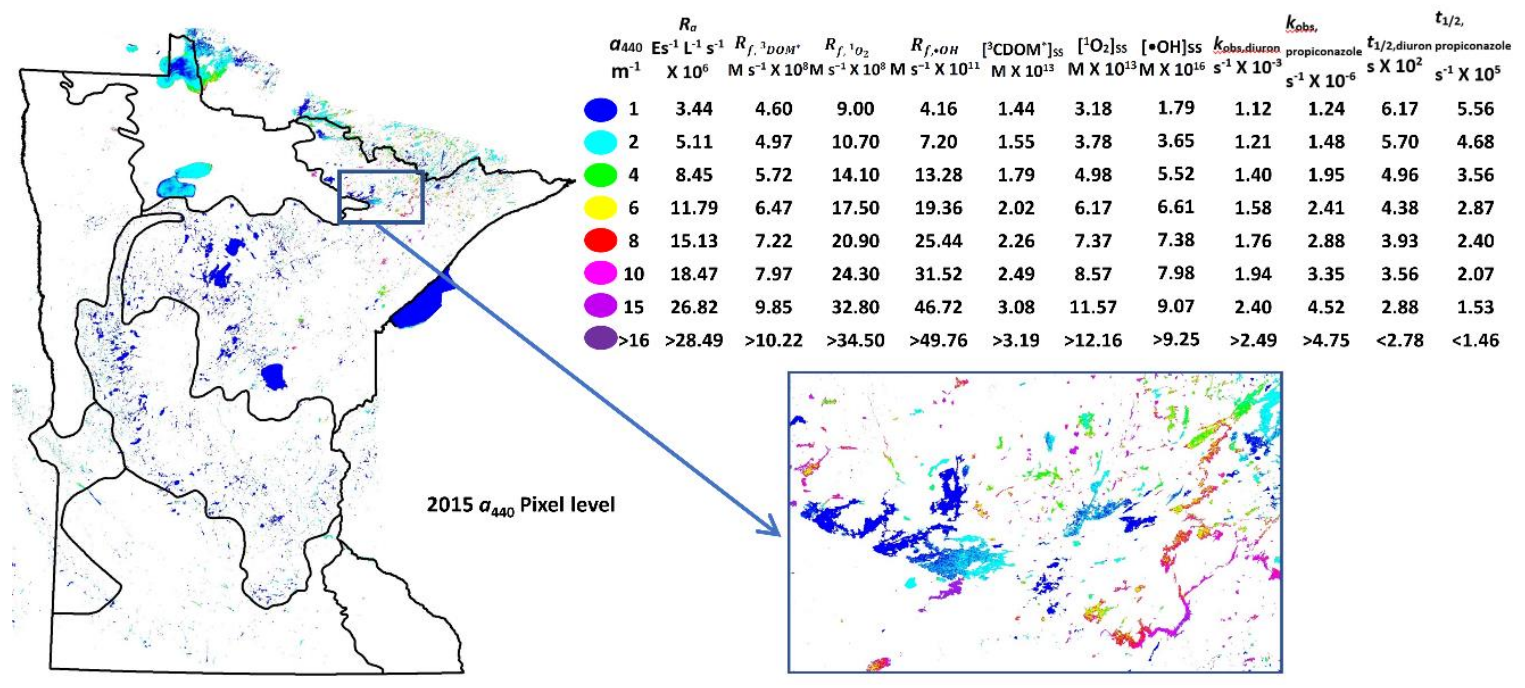

Figure S15. Distribution of estimated $R_{a}$, steady-state concentrations and formation rates of PPRIs, the first-order photodegradation rate constants and half-lives of pesticides in Minnesota lakes, based on pixel-level $a_{440}$ values in 2015. A zoom in of the NLF ecoregion in northeast Minnesota is shown in the inset figure. 


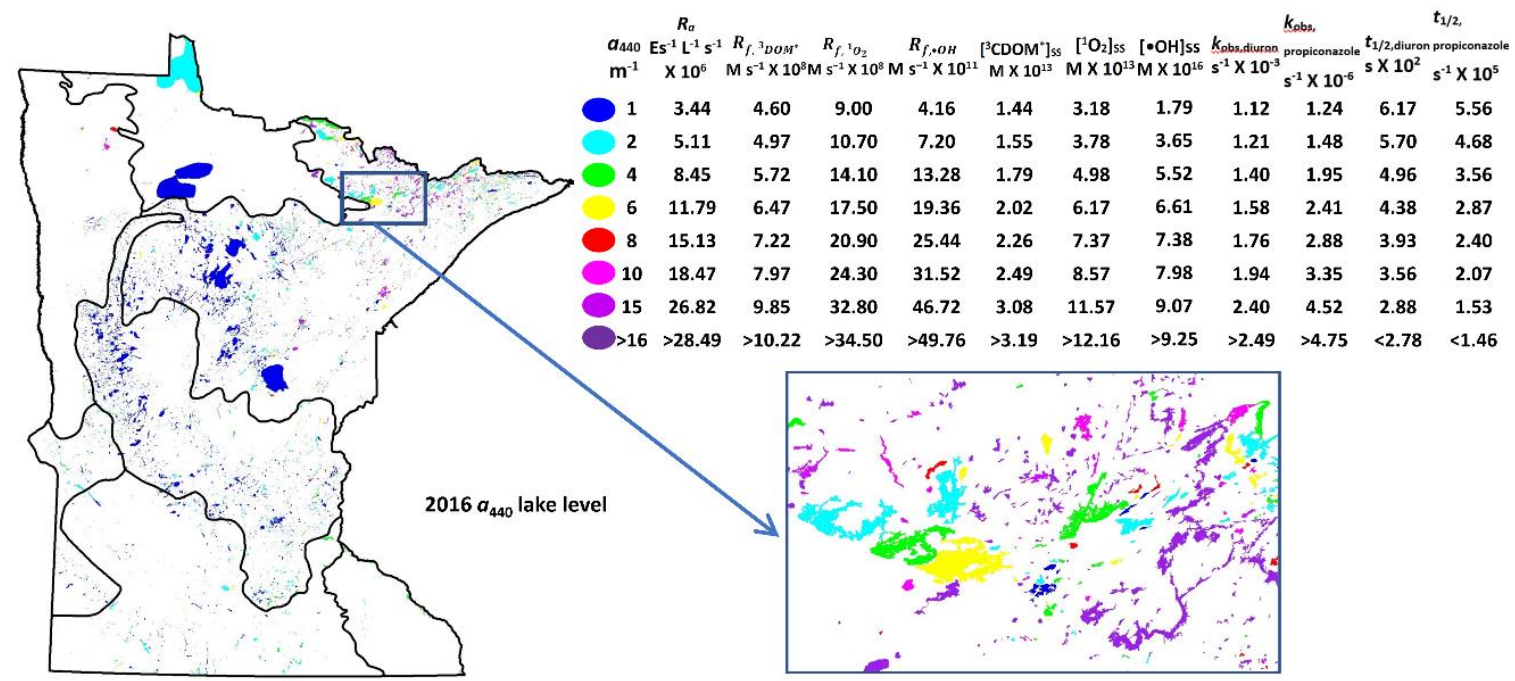

Figure S16. Distribution of estimated $R_{a}$, steady-state concentrations and formation rates of PPRIs, the first-order photodegradation rate constants and half-lives of pesticides in Minnesota lakes, based on lake average $a_{440}$ values in 2016. A zoom in of the NLF ecoregion in northeast Minnesota is shown in the inset figure.

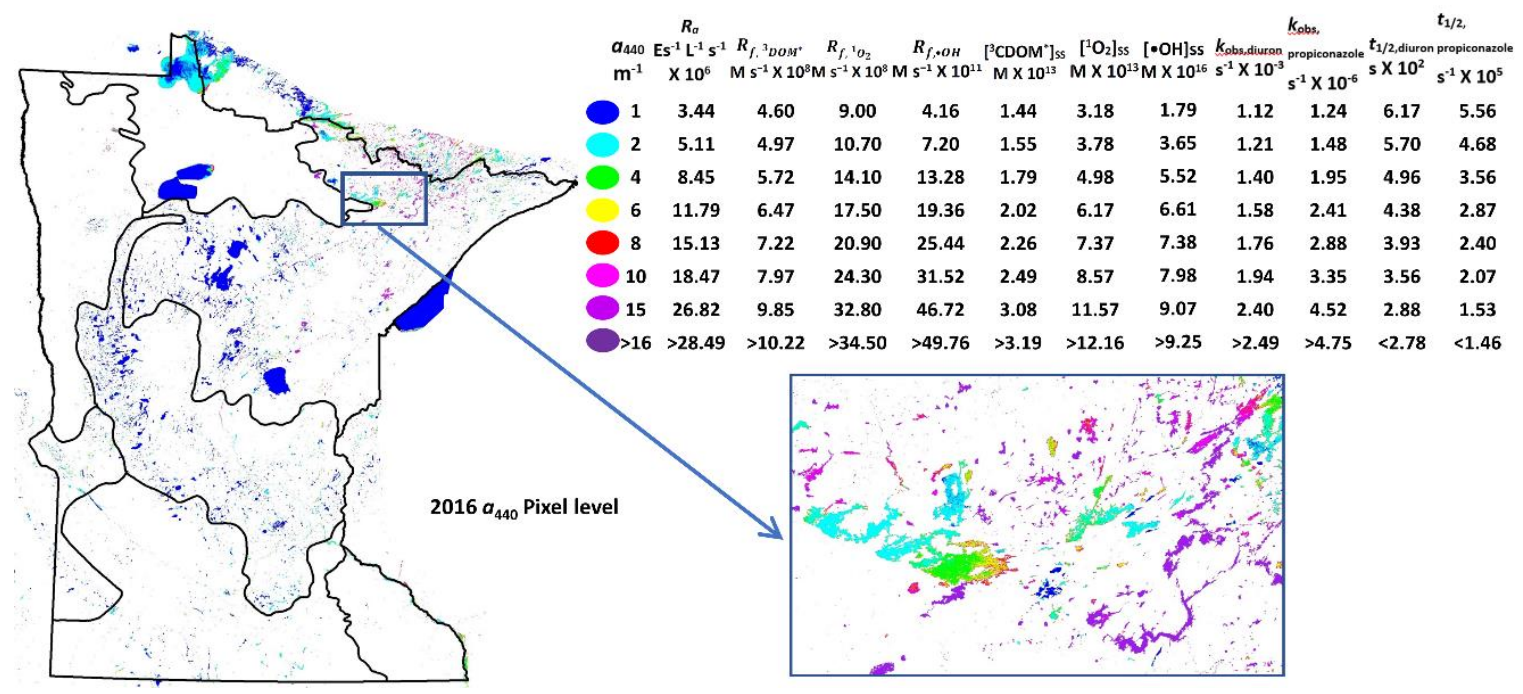

Figure S17. Distribution of estimated $R_{a}$, steady-state concentrations and formation rates of PPRIs, the first-order photodegradation rate constants and half-lives of pesticides in Minnesota lakes, based on pixel-level $a_{440}$ values in 2016. A zoom in of the NLF ecoregion in northeast Minnesota is shown in the inset figure. 


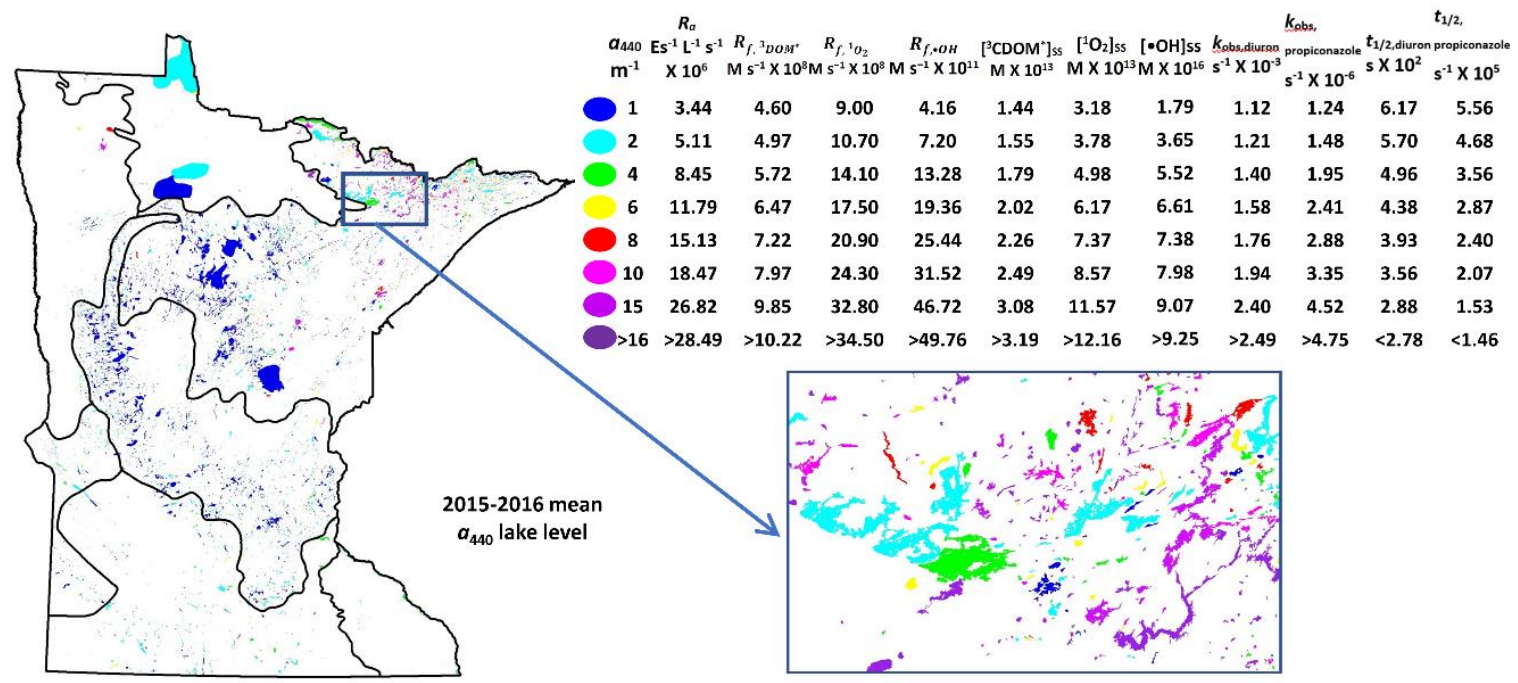

Figure S18. Distribution of estimated $R_{a}$, steady-state concentrations and formation rates of PPRIs, the first-order photodegradation rate constants and half-lives of pesticides in Minnesota lakes, based on average of lake average $a_{440}$ values in 2015 and 2016. A zoom in of the NLF ecoregion in northeast Minnesota is shown in the inset figure. 


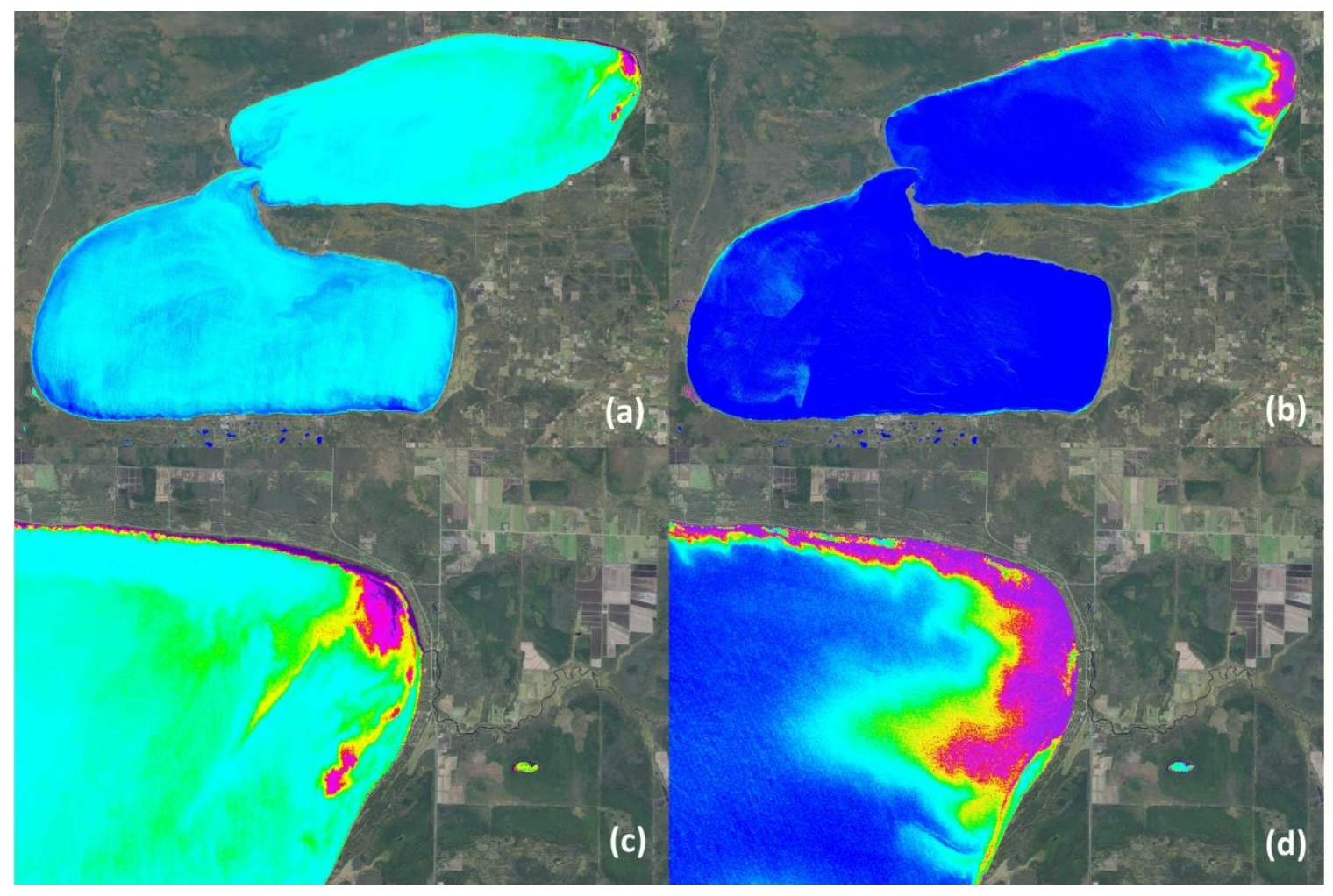

Figure S19. Pixel-level $a_{440}$ distribution in Upper and Lower Red Lake based on satellite imagery collected in (a) 2015 and (b) 2016. A zoom in of the Red Lake in 2015 and 2016 are shown in (c) and (d). Inflow of highly colored water from the Tamarack River at the northeast end of Upper Red Lake accounts for the high $a_{440}$ values in the eastern part of the lake. High values along the shorelines of both lakes likely reflect interference from bottom and emergent vegetation effects. 


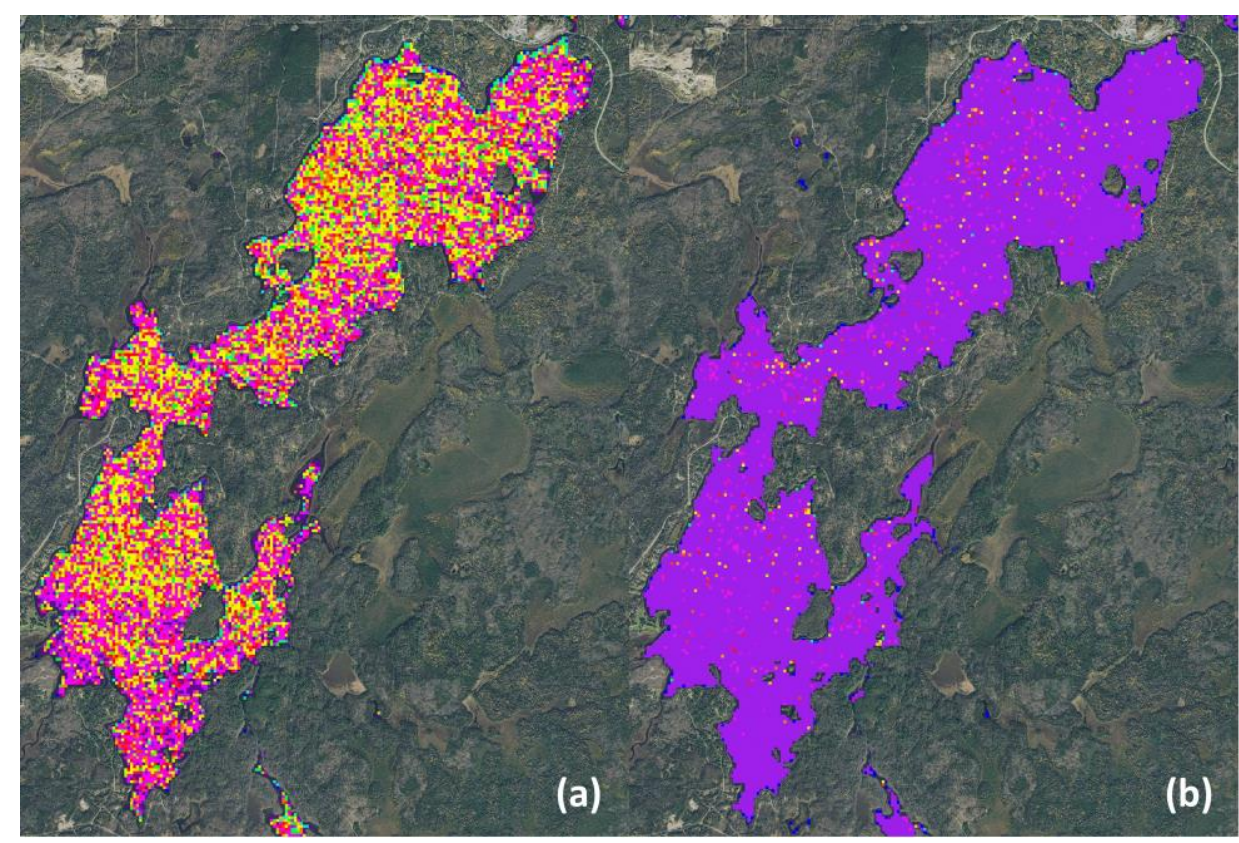

Figure S20. Pixel-level $a_{440}$ distribution in South and North White Iron Lake near Ely, Minnesota based on satellite imagery collected in (a) 2015 and (b) 2016. 

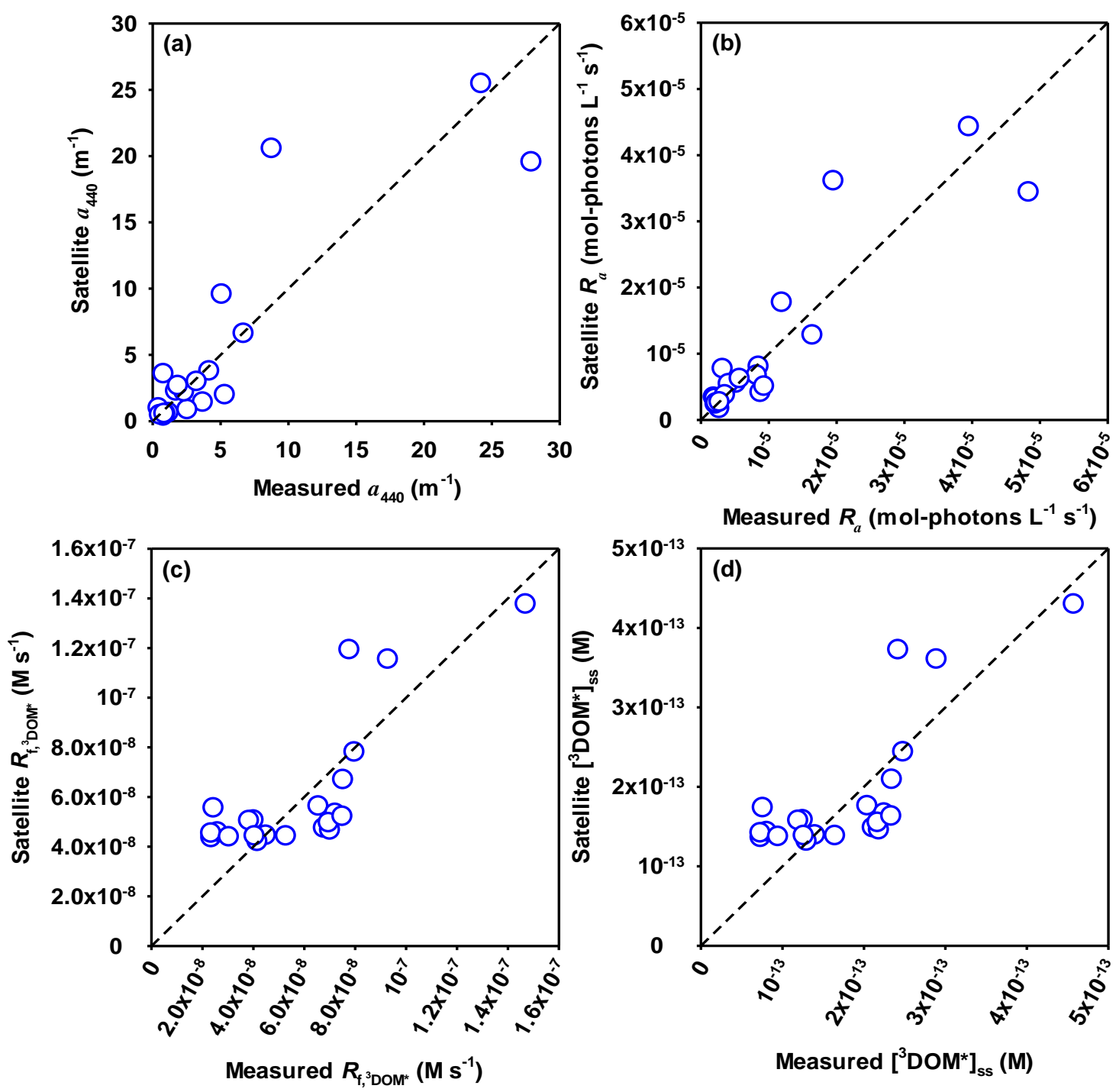

Figure S21. Comparison of estimated (satellite) and measured values of (a) $a_{440}$, (b) $R_{a}$; (c) $R_{f,{ }^{3} \mathrm{DOM}^{*}}$; and (d) $\left[{ }^{3} \mathrm{DOM}^{*}\right]_{\mathrm{ss}}$. The dashed line is a 1:1 line. Mississippi River and Upper Red \#2 (at the mouth of the Tamarack River) were excluded because the riverine flows create highly dynamic conditions that are not suitable for comparisons with relatively infrequent satellite imagery. 


\section{REFERENCES}

(1) Canonica, S.; Freiburghaus, M. Electron-Rich Phenols for Probing the Photochemical Reactivity of Freshwaters. Environ. Sci. Technol. 2001, 35 (4), 690-695. https://doi.org/10.1021/es0011360.

(2) Tratnyek, P. G.; Hoigné, J. Photooxidation of 2,4,6-Trimethylphenol in Natural Waters and Laboratory Systems: Kinetics of Reaction with Singlet Oxygen. $J$. Photochem. Photobiol. A Chem. 1992, 32, No. 1 (2), 220-221.

(3) Al Housari, F.; Vione, D.; Chiron, S.; Barbati, S. Reactive Photoinduced Species in Estuarine Waters. Characterization of Hydroxyl Radical, Singlet Oxygen and Dissolved Organic Matter Triplet State in Natural Oxidation Processes.

Photochem. Photobiol. Sci. 2010, 9 (1), 78-86.

https://doi.org/10.1039/b9pp00030e.

(4) Erickson, P. R.; Moor, K. J.; Werner, J. J.; Latch, D. E.; Arnold, W. A.; McNeill, K. Singlet Oxygen Phosphorescence as a Probe for Triplet-State Dissolved Organic Matter Reactivity. Environ. Sci. Technol. 2018, 52 (16), 9170-9178. https://doi.org/10.1021/acs.est.8b02379.

(5) Golanoski, K. S.; Fang, S.; Del Vecchio, R.; Blough, N. V. Investigating the Mechanism of Phenol Photooxidation by Humic Substances. Environ. Sci. Technol. 2012, 46 (7), 3912-3920. https://doi.org/10.1021/es300142y.

(6) McCabe, A. J.; Arnold, W. A. Seasonal and Spatial Variabilities in the Water Chemistry of Prairie Pothole Wetlands Influence the Photoproduction of Reactive Intermediates. Chemosphere 2016, 155, 640-647.

https://doi.org/10.1016/j.chemosphere.2016.04.078.

(7) McCabe, A. J.; Arnold, W. A. Reactivity of Triplet Excited States of Dissolved Natural Organic Matter in Stormflow from Mixed-Use Watersheds. Environ. Sci. Technol. 2017, 51 (17), 9718-9728. https://doi.org/10.1021/acs.est.7b01914.

(8) Zepp, R. G.; Schlotzhauer, P. F.; Sink, R. M. Photosensitized Transformations Involving Electronic Energy Transfer in Natural Waters: Role of Humic Substances. Environ. Sci. Technol. 1985, 19 (1), 74-81. https://doi.org/10.1021/es00131a008.

(9) Canonica, S.; Hellrung, B.; Wirz, J. Oxidation of Phenols by Triplet Aromatic Ketones in Aqueous Solution. J. Phys. Chem. A 2000, 104 (6), 1226-1232. https://doi.org/10.1021/jp9930550.

(10) Canonica, S.; Jans, U.; Stemmler, K.; Hoigne, J. Transformation Kinetics of Phenols in Water: Photosensitization by Dissolved Natural Organic Material and Aromatic Ketones. Environ. Sci. Technol. 1995, 29 (7), 1822-1831. https://doi.org/10.1021/es00007a020.

(11) Sharpless, C. M.; Aeschbacher, M.; Page, S. E.; Wenk, J.; Sander, M.; McNeill, K. Photooxidation-Induced Changes in Optical, Electrochemical, and Photochemical Properties of Humic Substances. Environ. Sci. Technol. 2014, 48 (5), 1-31. 
(12) Bodhipaksha, L. C.; Sharpless, C. M.; Chin, Y. P.; Sander, M.; Langston, W. K.; Mackay, A. A. Triplet Photochemistry of Effluent and Natural Organic Matter in Whole Water and Isolates from Effluent-Receiving Rivers. Environ. Sci. Technol. 2015, 49 (6), 3453-3463. https://doi.org/10.1021/es505081w.

(13) Maizel, A. C.; Remucal, C. K. The Effect of Probe Choice and Solution Conditions on the Apparent Photoreactivity of Dissolved Organic Matter. Environ. Sci. Process. Impacts 2017, 19 (8), 1040-1050. https://doi.org/10.1039/c7em00235a.

(14) Haag, W. R.; Hoigné, J.; Gassman, E.; Braun, A. M. Singlet Oxygen in Surface Waters - Part I: Furfuryl Alcohol as a Trapping Agent. Chemosphere 1984, 13 (56), 631-640. https://doi.org/10.1016/0045-6535(84)90199-1.

(15) McNeill, K.; Canonica, S. Triplet State Dissolved Organic Matter in Aquatic Photochemistry: Reaction Mechanisms, Substrate Scope, and Photophysical Properties. Environ. Sci. Process. Impacts 2016, Available online: http://dx.doi.org/10.1039/C6EM00. https://doi.org/10.1039/C6EM00408C.

(16) Page, S. E.; Arnold, W. A.; McNeill, K. Terephthalate as a Probe for Photochemically Generated Hydroxyl Radical. J. Environ. Monit. 2010, 12 (9), 1658-1665. https://doi.org/10.1039/c0em00160k.

(17) Page, S. E.; Arnold, W. A.; McNeill, K. Assessing the Contribution of Free Hydroxyl Radical in Organic Matter-Sensitized Photohydroxylation Reactions. Environ. Sci. Technol. 2011, 45 (7), 2818-2825. https://doi.org/10.1021/es2000694.

(18) Canonica, S.; Laubscher, H. U. Inhibitory Effect of Dissolved Organic Matter on Triplet-Induced Oxidation of Aquatic Contaminants. Photochem. Photobiol. Sci. 2008, 7 (5), 547-551. https://doi.org/10.1039/b719982a.

(19) Zeng, T.; Arnold, W. A. Pesticide Photolysis in Prairie Potholes: Probing Photosensitized Processes. Environ. Sci. Technol. 2013, 47 (13), 6735-6745. https://doi.org/10.1021/es3030808.

(20) Fang, X.; Mark, G.; Von Sonntag, C. OH Radical Formation by Ultrasound in Aqueous Solutions: Part I: The Chemistry Underlying the Terephthalate Dosimeter. Ultrason. Sonochem. 1996, 3 (1), 57-63. https://doi.org/10.1016/13504177(95)00032-1.

(21) Mark, G.; Tauber, A.; Laupert, R.; Schuchmann, H.-P.; Schulz, D.; Mues, A.; von Sonntag, C. OH-Radical Formation by Ultrasound in Aqueous Solution - Part II: Terephthalate and Fricke Dosimetry and the Influence of Various Conditions on the Sonolytic Yield. Ultrason. Sonochem. 1998, 5 (2), 41-52.

(22) Buxton, G. V.; Greenstock, C. L.; Helman, W. P.; Ross, A. B. Critical Review of Rate Constants for Reactions of Hydrated Electrons, Hydrogen Atoms and Hydroxyl Radicals $(\cdot \mathrm{OH} / \cdot \mathrm{O}-$ in Aqueous Solution. J. Phys. Chem. Ref. Data 1988, 17 (2), 513-886. https://doi.org/10.1063/1.555805. 
(23) Westerhoff, P.; Mezyk, S. P.; Cooper, W. J.; Minakata, D. Electron Pulse Radiolysis Determination of Hydroxyl Radical Rate Constants with Suwannee River Fulvic Acid and Other Dissolved Organic Matter Isolates. Environ. Sci. Technol. 2007, 41 (13), 4640-4646. https://doi.org/10.1021/es062529n.

(24) Grebel, J. E.; Pignatello, J. J.; Mitch, W. A. Effect of Halide Ions and Carbonates on Organic Contaminant Degradation by Hydroxyl Radical-Based Advanced Oxidation Processes in Saline Waters. Environ. Sci. Technol. 2010, 44 (17), 68226828. https://doi.org/10.1021/es1010225.

(25) Yang, Y.; Pignatello, J. J.; Ma, J.; Mitch, W. A. Comparison of Halide Impacts on the Efficiency of Contaminant Degradation by Sulfate and Hydroxyl RadicalBased Advanced Oxidation Processes (AOPs). Environ. Sci. Technol. 2014, 48 (4), 2344-2351. https://doi.org/10.1021/es404118q.

(26) Leifer, A. The Kinetics of Environmental Aquatic Photochemistry; 1989; Vol. 61. https://doi.org/10.1021/ac00190a728.

(27) O’Connor, M.; Helal, S. R.; Latch, D. E.; Arnold, W. A. Quantifying PhotoProduction of Triplet Excited States and Singlet Oxygen from Effluent Organic Matter. Water Res. 2019, 156, 23-33. https://doi.org/10.1016/j.watres.2019.03.002.

(28) McKay, G.; Huang, W.; Romera-Castillo, C.; Crouch, J. E.; Rosario-Ortiz, F. L.; Jaffé, R. Predicting Reactive Intermediate Quantum Yields from Dissolved Organic Matter Photolysis Using Optical Properties and Antioxidant Capacity. Environ. Sci. Technol. 2017, 51 (10), 5404-5413. https://doi.org/10.1021/acs.est.6b06372.

(29) Olmanson, L. G.; Page, B. P.; Finlay, J. C.; Brezonik, P. L.; Bauer, M. E.; Griffin, C. G.; Hozalski, R. M. Regional Measurements and Spatial/Temporal Analysis of CDOM in 10,000+ Optically Variable Minnesota Lakes Using Landsat 8 Imagery. Sci. Total Environ. 2020, 138141. https://doi.org/10.1016/j.scitotenv.2020.138141. 